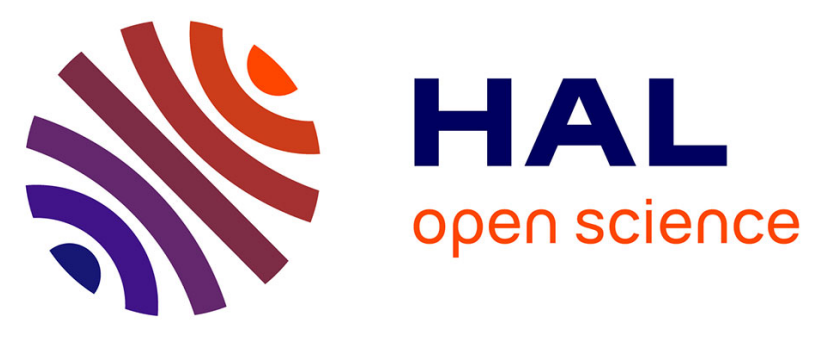

\title{
Nanotechnologies for Intracellular Protein Delivery: Recent Progress in Inorganic and Organic Nanocarriers
}

Sarah Le Saux, Anne Aubert-pouëssel, Lyria Ouchait, Khaled Elhady

Mohamed, Pierre Martineau, Laurence Guglielmi, Jean-marie Devoisselle, Philippe Legrand, Joël Chopineau, Marie Morille

\section{To cite this version:}

Sarah Le Saux, Anne Aubert-pouëssel, Lyria Ouchait, Khaled Elhady Mohamed, Pierre Martineau, et al.. Nanotechnologies for Intracellular Protein Delivery: Recent Progress in Inorganic and Organic Nanocarriers. Advanced Therapeutics, 2021, pp.2100009. 10.1002/adtp.202100009 . hal-03210215

\section{HAL Id: hal-03210215 \\ https://hal.science/hal-03210215}

Submitted on 27 Apr 2021

HAL is a multi-disciplinary open access archive for the deposit and dissemination of scientific research documents, whether they are published or not. The documents may come from teaching and research institutions in France or abroad, or from public or private research centers.
L'archive ouverte pluridisciplinaire HAL, est destinée au dépôt et à la diffusion de documents scientifiques de niveau recherche, publiés ou non, émanant des établissements d'enseignement et de recherche français ou étrangers, des laboratoires publics ou privés. 
Nanotechnologies for intracellular protein delivery: recent progress in inorganic and organic nanocarriers.

Sarah LE SAUX ${ }^{1}$, Anne AUBERT-POUËSSEL ${ }^{1}$, Lyria OUCHAIT $^{1}$, Khaled Elhady MOHAMED $^{1}$, Pierre MARTINEAU ${ }^{2}$, Laurence GUGLIELMI $^{2}$, Jean-Marie DEVOISSELLE $^{1}$, Philippe LEGRAND ${ }^{1}$, Joël CHOPINEAU ${ }^{1}$, Marie MORILLE ${ }^{1 *}$.

${ }^{1}$ ICGM, Univ Montpellier, ENSCM, CNRS, Montpellier, France;

${ }^{2}$ IRCM, Univ Montpellier, Inserm, Montpellier, France;

* Corresponding author: marie.morille@ umontpellier.fr; ORCID: 0000-0001-8224-7346 


\begin{abstract}
From recombinant insulin to monoclonal antibodies, proteins have become essential drugs in the therapeutic arsenal and respond to a broad spectrum of diseases. While commercially available proteins have an extracellular activity, intracellular targets could be a powerful alternative to treat many pathologies such as cancers, immune disorders, but also infectious diseases. Nevertheless, to enter the cell and reach the target organelle or molecule, therapeutic proteins have to face numerous barriers (internalisation, endosomal escape, cytoplasm trafficking). In view of the fragile nature and high heterogeneity of proteins in term of size, charge and hydrophilic/lipophilic profile, the development of an efficient cytoplasm delivery system remains a long-standing challenge. The discovery of several gene editing tools (CRISPR-Cas9, TALEN, or other ZFP) has particularly boosted this research field in the last decade. Among delivery systems that can be used in vivo, some synthetic nanovectors offer interesting opportunities. In this review, we decided to describe the most recent progress in development of inorganic (silica, gold, phosphate, metal organic framework, carbon nanoparticles) as well as organic (polymer, lipid) nanoparticles both applied to intracellular protein delivery.
\end{abstract}

Key words: cytoplasmic protein delivery - therapeutic protein - inorganic nanoparticles - lipid nanoparticles - polymer nanoparticles - CRISPR Cas9 


\section{Introduction}

Protein-based therapies are now becoming more and more present in the therapeutic arsenal, from 130 therapeutic proteins approved by the Food and Drug Administration (FDA) in 2008 [1] to 239 in 2017 [2]. These therapeutic proteins are used to treat a large panel of diseases (cancers, immune disorders, infections, etc). The market of protein therapeutics, mainly dominated by monoclonal antibodies, is estimated at $10 \%$ (worldwide sales) of the entire pharmaceutical market, and promises to continue growing in the future [3]. Compared to synthetic molecules, proteins possess the ability to act at lower concentration with high substrate specificity therefore leading to fewer side effects [4].

Even if two-third of the proteome is supposed to be found inside the cells, where proteins assemble one another in a complex and precise way to mediate cellular function (or dysfunction), most marketed proteins present an extracellular activity (targeting extracellular membrane receptors, or soluble proteins) [5]. However, the use of intracellular protein delivery could bring a solution for various applications: (i) supplying an extrinsic protein if the intrinsic protein is missing or ineffective (ex : enzyme defect in lysosomal storage disease (such as glucocerebrosidase in Gaucher disease, etc), (ii) introducing a previously inexistent function (ex: to control gene edition with Clustered Regularly Interspaced Short Palindromic Repeat (CRISPR), involving caspase 9 (Cas9) protein (CRISPR-Cas9), zinc-finger proteins (ZFPs), transcription activator-like effectors (TALEs) or (iii) interfering with protein-protein interactions and modifying intracellular pathways (ex: intrabodies or other non antibody protein scaffolds $[6,7])$. Altogether, these needs have called for the development of efficient protein delivery systems to the cytoplasm.

The complexity of these macromolecules, which is the basis for their high efficiency and specificity, becomes a critical point for their pharmaceutical development. Given the diversity of such molecules, there is no universal solution, and protein stabilisation must be thought off in a case-by-case approach [8]. Moreover, to be able to reach intracellular targets, additional efforts have to be made to: (1) cross hydrophobic and negatively charged cell membranes reinforced in the intracellular side by a dense actin network, (2) escape from the endosomes to avoid lysosomal degradation, (3) traffic in cell cytoplasm toward its target and (4) enter the targeted organelle if required (such as nucleus or mitochondria), depending on the protein mode of action (Figure 1). To supply a protein into the cytoplasm, a first approach could consist of over-expressing proteins by transfecting cells. This strategy is now well documented, but from a therapeutic point of view, the genetic manipulation of cells requires 
ex vivo modifications and entails unequal transfection yields depending on the transfected cell types. Another promising strategy is based on bringing proteins or peptides of interest into a cell, without impacting the genetic material of such cells. Physical-based techniques like electroporation [9], microinjection [10] and sonoporation [11] have been explored to this end. Engineered nanodevices, such as silicon nanoneedles (nanowire, nanostraw or nanotubes) were recently developed for intracellular delivery of a wide range of therapeutics including biomolecules (proteins, nucleic acids) [12-15]. Nevertheless, although they can prove effective, these physical-based methods are mainly dedicated to in vitro or ex-vivo applications and do not provide a convenient way to deliver a therapeutic protein in vivo, or would be limited to first layers of cells in the tissue (regarding nanoneedles scale) [16].

Regarding the complexity of therapeutic proteins, several nanoscale synthetic systems (lipid or polymer cationic complexes, nanoparticles, micelles, etc.) have been developed (Figure 2), but to date, it is difficult to identify a versatile system able to encapsulate and deliver a wide range of protein into different cell source. In this review, we have chosen to focus on recent strategies developed to deliver functional intracellular proteins while maintaining their chemical and physical stability. As recent progress has been made in the field of inorganic nanocarriers, we have chosen to describe these systems in parallel to organic carriers more often encountered for biological applications. We preferred to rule out cell-penetrating peptides (CPPs) as they have been thoroughly reviewed recently [17, 18], as well as the use of fusion proteins or other irreversible protein modifications. This review therefore describes mainly the physical entrapment of proteins while avoiding studies reporting chemical encapsulation via covalent linkage. A specific focus was given to systems allowing delivery of gene-editing tools, and especially CRISPR-Cas9 associated or not with guide RNA (sgRNA) (forming RiboNucleoProtein (RNP) complexes) and /or template DNA. Special attention was also paid to systems which had potential for in vivo administration. The first part of this review is dedicated to inorganic nanocarriers of mineral, metallic, carbonbased or of hybrid nature for intracellular protein delivery. The second part focuses on describing organic nanocarriers based on polymers and lipids. 


\section{Inorganic nanocarriers}

In general, in the literature, inorganic nanocarriers are represented by solid nanoparticles usually derived from metallic compounds, but also from mineral compounds such as silica or phosphate. The advantages of such inorganic carriers over their organic counterparts rely on: (i) the fact that synthesis can be performed in aqueous medium, (ii) some specific properties/chemical reactivity which could allow alternative targeting mechanism (magnetic properties), (iii) efficient imaging properties (optical properties) and (iv) triggered release/dissociation in response to external environment stimuli such as hyperthermia (photothermal properties). The following section is a non-exhaustive description of recent advances in using such nanocarriers for protein intracellular delivery.

\subsection{Phosphate-based nanoparticles}

As main mineral phase of most biological hard tissues, calcium phosphate $(\mathrm{CaP})$ is highly biocompatible. This $\mathrm{pH}$-sensitive inorganic material, which degrades or dissolves at acidic $\mathrm{pH}$, has been used for decades in the form of nanoparticles (CaP NPs) for the intracellular delivery of nucleic acids as well as small molecules. Recently, Zeng et al. [19] synthesized, by a precipitation method, $100 \mathrm{~nm}$ CaP NPs encapsulating model proteins (Bovine Serum Albumin (BSA), Green Fluorescent Protein (GFP), Killer Red protein) with a high reported protein loading capacity $(29 \%(\mathrm{w} / \mathrm{w}))$. Flow cytometry analysis revealed a timedependant endocytosis of GFP-CaP NPs into hepatocyte cell line HepG2. This fluorescence staining was dispersed in the cytosol, confirming an escape of GFP from the endolysosomal pathway. The authors reported the ability of CaP NPs to trigger a proton sponge effect allowing their release from endosomes as encountered with cationic polymers such as poly (ethylenimine) (PEI) [20]. Besides nanospheres, CaP nanorods (33 - $35 \mathrm{~nm}$ length; 4 - $5 \mathrm{~nm}$ diameter) were recently developed and coated with polyacrylate to complex endoribonuclease A (RNase A, 13.7kDa), having a cytotoxic effect once transferred in Hela cell cytoplasm [21]. The authors reported efficient biological activity of RNase by tracking cell cytotoxicity in Hela cells. A dose-dependent increase in cytotoxicity was indeed observed when RNase was complexed with nanorods, whereas this was not observed with free RNase or nanorods only. $\mathrm{CaP}$ nanorods could therefore be used for in vitro protein delivery, even if this system has to be evaluated in different cell types. Nevertheless, it should be noted that, as proteins are adsorbed on nanorods surface, no protein protection is provided, and in vivo use could be limited by this point. The advantage of using CaP NPs to complex proteins and allow their intracellular delivery mainly relies on their biodegradability and biocompatibility. If their 
main drawback (i.e. ability to form nanoscaled reproducible aggregates which are stable in vivo) could be circumvented, these systems could, without a doubt, represent an interesting option in the future. A promising option could be their association with other organic particles, as it has been successfully done with liposome for nucleic acid release for example [22].

This strategy was evaluated with other $\mathrm{pH}$ sensible materials: magnesium phosphate NPs (MgP NPs). MgP NP were associated with cationic lipids (DOTAP), thus creating lipid coated magnesium phosphate nanoparticles (LPPs) [23]. LPPs were loaded with catalase (CAT), a ubiquitous anti-oxidant enzyme. In serum-free medium, CAT-loaded LPPs were able to fully counteract viability loss of MCF-7 cells due to $\mathrm{H}_{2} \mathrm{O}_{2}$ test in contrast to free catalase treatment (recovery of $50 \%$ of the viability), but the same effect was observed with CAT-loaded DOTAP liposomes. In contrast, when serum was present in the medium, a slight advantage in reactive oxygen species (ROS) protection was noticed with CAT-LPPs compared to CAT liposomes only. Importantly, similarly to CaP NPs, the use of cationic lipids is a necessity to provide at least cell internalisation. Moreover, it was reported that LPPs alone induced cellular toxicity, probably due to the presence of positively charged DOTAP.

To summarize, in regards to their evocated drawbacks, phosphate NP based formulations are an interesting building blocks which could probably be improved with pharmaceutical development.

\subsection{Silica-based nanoparticles}

\subsubsection{Mesoporous silica nanoparticles}

For nearly 20 years now, mesoporous silica nanoparticles (MSNPs) have warranted increasing interest for drug delivery, mainly owing to their high drug loading capacity, stiff framework, precise pore structure, easily controllable morphology, and tuneable surface chemistry [24-26]. Several parameters could be adjusted to influence release profile of encapsulated therapeutics (pore size, surface area, symmetry and surface modification).

The recent development of MSNPs with increased pore size opened the way for the association/incorporation of macromolecules such as proteins. High pore size is however generally associated to a fast release profile (burst release). In this context, non-covalent bindings, such as electrostatic interactions used in the following studies, are therefore required to control protein association and release. MSNPs functionalized with nickel moieties were used to complex, by electrostatic interactions, His-tagged $\beta 4$ proteasome subunits $(29 \mathrm{kDa})$ into 25-30nm pores with the goal to destruct intracellular tau aggregates in HEK293-derived 
cell line over expressing the longest central nervous system isoform of human tau [27] (Table 1). $\beta 4$ proteasome subunit loaded MS NPs were internalised and distributed to the cytosol after endosomal escape and were able to reach their target. In vitro tests showed proteasome loaded MSNPs degraded the overexpressed tau in cells more efficiently (in regards to native proteasomes, which resulted in decreased levels of pathological truncated tau. This study holds great promise for the treatment of Alzheimer's disease but also of all pathologies involving protein misfolding and aggregation such as amyotrophic lateral sclerosis, Parkinson's disease, and Huntington's disease.

Aiming to carry high weight molecular proteins, and relying on pore structure to control protein delivery, Xu et al. [28] synthesized MSNPs with a core-cone structure (MSNCC) (Table 1). Exposing pore size of $45 \mathrm{~nm}$ and a diameter of $220 \mathrm{~nm}$, MSN-CC demonstrated a high loading capacity of both immunoglobulin $\mathrm{G}(\mathrm{IgG})(150 \mathrm{kDa}, 560 \mu \mathrm{g} / \mathrm{mg}$ of MSN-CC) and $\beta$-galactosidase ( $\beta$-gal) tetramer (480 kDa, $190 \mu \mathrm{g} / \mathrm{mg}$ of MSN-CC). MSN$\mathrm{CC}$ were subsequently shown to efficiently deliver rhodamine B isothiocyanate labelled IgG (RITC-IgG) inside N2A neuroblastoma cell line as well as biologically active $\beta$-gal, as assessed by X-gal staining, present at high levels in $90 \%$ of treated cells. With the same goal, Amine-functionalised Hollow Dendritic Mesoporous Silica NanosPheres (A-HDMSNPs) were synthesized to carry IgG and $\beta$-gal [29] (Figure 2). A-HDMSNPs exposed a diameter of $360 \pm 37 \mathrm{~nm}$ and large mesopores of $20 \mathrm{~nm}$ in the shell. Electrostatic interactions were evidenced to be a key parameter for protein interaction, as assessed by both $\operatorname{IgG}$ and $\beta$ galactosidase association with amine functionalised A-HDMSNPs in comparison to nonfunctionalised HDMSNPs (protein loading of $391 \pm 16 \mu \mathrm{g} / \mathrm{mg}$ vs. $162 \pm 19 \mu \mathrm{g} / \mathrm{mg}$ for $\beta$-gal and $658 \pm 45 \mu \mathrm{g} / \mathrm{mg}$ vs. $416 \pm 53 \mu \mathrm{g} / \mathrm{mg}$ for IgG). Amine-functionalised A-HDMSNPs allowed a 39-fold increase in CHO-K1 cellular uptake of RITC- $\beta$-gal vs. free RITC- $\beta$-gal. Finally, enzymatic activity of intracellularly delivered $\beta$-gal was assessed through X-gal staining. Altogether, these studies established mesoporous silica nanoparticles as efficient nanocarriers even for the intracellular delivery of high molecular weight proteins.

To allow environment inducible responsiveness and favour release in tumour cell cytoplasm exposing high level of GSH, MSNP were synthesized with glutathione (GSH)dependent sensitive moieties (disulfide bonds) incorporated in DenDritic Mesoporous Organosilica NP (DDMONPs) [30] (Table 1). To trigger endosomal escape, DDMONPs were also functionalised with PEI, and the association of RNase A (positively charged at physiological $\mathrm{pH}$ ) to cationic $\mathrm{PEI}$, was made possible by converting positively charged lysine residues of RNAse A into negatively charged carboxylate groups (RNase A - Aco) with cis- 
aconitic anhydride (Aco). Thereby formed systems (DDMONPs-PEI) displayed a loading efficiency with RNase A-Aco of $426 \pm 15 \mu \mathrm{g} / \mathrm{mg}$. In vitro, a GSH concentration-dependent release was confirmed ( $\sim 57 \%$ released at $48 \mathrm{~h}$ for $1 \mathrm{mM}$ vs $97 \%$ for $10 \mathrm{mM} \mathrm{GSH}$ containing release media). A higher cytotoxic effect was observed after DDMONPs-PEI/RNase A-Aco treatment of cancer cells (B12F0 melanoma cells) compared to healthy cells (HEK293 human embryonic cell line). Surprisingly, efficient protein intracellular delivery in cancer cells was achieved, while reducing cytotoxicity was observed in normal cells exposing lower cytoplasmic glutathione level. Nevertheless, once again, protein association performed on the surface of particles could lead to degradation by protease if used in vivo, and cytotoxicity associated with PEI could also be an issue and a reason why such systems have, to our knowledge, not been evaluated in vivo yet.

Still focusing on synthesizing environment-responsive systems, diselenide-bondexposing organosilica moieties were included into silica architecture to synthesize biodegradable MSNPs with redox responsiveness [31] (Table 1). Resulting MSNPs with 8-10 $\mathrm{nm}$ internal diameter pores were able to entrap RNase A via electrostatic interactions (270 $\mu \mathrm{g} / \mathrm{mg})$ and to release their content upon exposure to oxidative $\left(\mathrm{H}_{2} \mathrm{O}_{2}\right)$ or reducing conditions (high GSH concentrations). These systems were coated with cancer cell-derived membranes (CM) issued from HeLa cells extrusion process. Interestingly, an increase in HeLa cell internalisation was evidenced when MSNPs were coated with CM, whereas a decrease in internalisation was observed into Raw264.7 macrophages using the same RNase A-MSNPs formulation. The authors explain this macrophage escape by the presence of CD47 membrane protein, brought by the CM coating, acting as a "do not eat me" signal. This macrophage escape translated in vivo to a significant increase in blood half-life following intravenous (IV) injection into to HeLa tumour bearing nude mice. A majority of RNAse-loaded MS NPs (over $40 \%$ ), coated or not with CM, were found to accumulate in the liver. Nevertheless, a significant increase in tumour accumulation was evidenced with CM coated MSNPs vs. non coated, and coated NPs significantly decreased tumour growth after 28 days of treatment (injections every 4 days). This system therefore represents a promising candidate for intracellular protein delivery, and is, to date, one of the only nanocarrier based on silica used in vivo for protein intracellular delivery.

\subsubsection{Solid silica nanoparticles}

Non-porous solid silica NPs have less been explored but have shown interesting results to achieve intracellular delivery. Instead of relying on electrostatic interactions to 
control protein loading and release, another strategy could be to modify silica NP with hydrophobic groups to allow interaction with hydrophobic domains of proteins. Bale et al. first demonstrated that modified with a hydrophobic coating ( $n$-octadecyltrimethoxysilane), such NPs were able to deliver RNase A and induce cell death in cancerous cells (MCF-7) and rat neural stem cells [32]. Recently, smooth silica nanoparticles (SSNPs) and rough silica nanoparticles (RSNPs) (SSNPs decorated with $20 \mathrm{~nm}$ silica NPs) (Table 1) were functionalised with hydrophobic octadecyl groups to evaluate the impact of surface roughness and hydrophobicity on (i) protein adsorption capacity, (ii) release behaviour, (iii) cellular uptake and (iv) endo-lysosome escape efficiency [33]. Both surface roughness and hydrophobic modifications were shown to enhance RNase A adsorption capacity (from $7.3 \mu \mathrm{g}$ to $20.7 \mu \mathrm{g}$ per mg NPs) leading to a sustained release reaching $100 \%$ at $72 \mathrm{~h}$ (PBS, pH7.4). Interestingly, only surface hydrophobic modifications facilitated endosomal escape. Consequently, hydrophobic octadecyl-functionalised RSNPs were the most efficient system for RNase A delivery, causing significant cell death (> $75 \%$ ) in both human breast cancer (MCF-7) and rat neural stem cell lines.

\subsubsection{Silica vesicles}

In contrast to solid silica NPs where only the outward surface can be utilised, silica vesicles (SVs) with tunable aperture size (from 6 to $34 \mathrm{~nm}$ ) on thin shells (Table 1) have been explored to carry proteins. SVs with entrance pore of $6 \mathrm{~nm}$ enabled RNase A loading as well as complete sustained release over $70 \mathrm{~h}$ at $37{ }^{\circ} \mathrm{C}$ in cell culture media (Dulbecco's Modified Eagle Medium:Nutrient Mixture F-12) [34]. The same formulation led to a convincing decrease in SCC25 cancerous cell viability of $70 \%$ after $72 \mathrm{~h}$ of treatment with RNase-loaded SVs, whereas no decrease was observed with unloaded SVs.

To conclude concerning silica NPs for protein loading, some convincing in vitro results can be found in the literature, especially recently on high molecular weight proteins. To date, based on our knowledge, only one team has evaluated silica based protein-NPs in vivo [31] with a cancer cell derived membrane coating, and poor biodistribution was showed. Evidencing in vivo efficiency is probably the main challenge for protein intracellular delivery based on silica NPs today, but in regards to the active research in the field, such studies will surely emerge soon. Moreover, silica was recently used as part of a smart system composed of Au NP aggregates and an endosomal disruptive polymer [35]. This hybrid carrier, developed in the following section, is among the most promising option currently investigated for CRISPR Cas 9 delivery. 


\subsection{Gold nanoparticles}

Associating photo-thermal, optical, chemical reactivity properties, fine control of size and shape and bio-compatibility, gold nanoparticles (Au NPs) have now been extensively investigated for drug delivery [36]. Rottello's group was one of the first to investigate functionalised Au NPs for protein intracellular delivery [37] (Figure 3A). Au NPs core (13 $\mathrm{nm}$ ) were functionalised with HKRK (a small cationic peptide) leading to Au NPs exposing a positive charge (zeta potential, $+32 \mathrm{mV}$ ). When associated to the particles, high molecular weight negatively charged enzyme $\beta$-gal was able to cross cell membrane, enter different cell types (COS-1, MCF7, C2C12) and carry out its biologic activity without inducing noticeable cytotoxicity. Nevertheless, it appeared that the majority of carriers were entrapped in endosomes. The same team therefore developed NanoParticle-Stabilised Capsules (NPSCs): aforementioned Au NPs functionalised with HKRK were used to stabilise linoleic acid-based nano-emulsions, thus forming so-called Pickering emulsions [38] (Figure 3B). These $130 \mathrm{~nm}$ NPSCs allowed the delivery of biologically active caspase-3 (Cas3) (35 kDa) to HeLa cells, inducing subsequent apoptosis. NPSCs were then adapted to vectorize high molecular weight $\beta$-gal protein [39]. Interestingly, live cell imaging demonstrated that NPSCs delivered their protein cargo through a direct 'membrane-fusion' mechanism, avoiding endosomal entrapment [38]. The same group recently reported interesting results with $2 \mathrm{~nm} \mathrm{Au} \mathrm{NP}$ functionalised with arginine-presenting ligands (ArgNPs) to electrostatically interact with proteins presenting an oligo-glutamic acid negative tag (E-tag) in their C-term ends [40] (Figure 3C). Hence, via these carboxylate-guanidinium electrostatic interactions, complex hierarchical assemblies of ArgNPs and five types of E-tagged proteins with different molecular weights, isoelectric points (prothymosin- $\alpha$ (PTMA), GFP, granzyme A, Cre recombinase, histone $2 \mathrm{~A}(\mathrm{H} 2 \mathrm{~A})$ were formed generating $350 \mathrm{~nm}$ range protein-ArgNPs structures. ArgNPs were used for the cytosolic delivery of CRISPR Cas9-RNP complexes into mammalian cells (Hela, HEK293, Raw264.7 cells lines) [41] (Figure 5, Table 5). This assembly led to $90 \%$ Cas9-RNP entry in the cytosol and nucleus of HeLa cells. Time-lapse imaging of Cas9-RNP-ArgNP complexes delivery showed a rapid release into the cytosol and subsequently to the nucleus. This allowed the achievement of up to $\sim 30 \%$ gene editing efficiency (non-homologous end-joining (NHEJ), Figure 5) of both the Adeno-associated virus integration site (AAVS1) and the tumor suppressor Phosphatase and TENsin homolog (PTEN) genes in vitro. In vivo, the system achieved more than $8 \%$ gene KO (by NHEJ) for 
PTEN gene in macrophages in the liver and spleen after ArgNPs systemic delivery [42]. In light of these results, it seems promising as vector for in vivo RNP delivery. Nevertheless, the 4 required injections necessary to reach this result raise the question of bioaccumulation risk associated with AuNP and their long-term fate.

Cas9-RNP delivery was also evaluated by Lee et al. who developed CRISPR/Cas9based therapeutics able to correct gene mutations via homology-directed repair (HDR) [35] (Figure 3F). For this, $15 \mathrm{~nm}$ AuNPs were functionalised with 5' thiol modified singlestranded DNA to complex the donor DNA template through complementary base-pairing. Cas9-RNP complexes were then added to the construct forming AuNP-donor DNA-Cas9RNP. Finally, this system was coated with silica to increase stability and with an endosomal disruptive polymer (poly $(N-[N-(2$-aminoethyl)-2-aminoethyl $]$ aspartamide) (PAsp(DET)) to allow the release of NPs into the cytoplasm. In vitro HDR experiments were performed on HEK293 cells expressing blue fluorescent protein (BFP). CRISPR-AuNPs associated to a single-stranded donor oligonucleotidethat allow convertion of BFP gene into the GFP gene, and a gRNA able to cut the BFP gene, were shown to induce GFP expression via HDR in $11.3 \%$ of BFP-HEK. In vitro CRISPR-AuNPs delivery efficiency was then investigated in a wide range of primary cells including myoblasts from $\mathrm{mdx}$ mice (model for Duchenne muscular dystrophy (DMD)). HDR efficiency was between 3-4 \%, this could be seen as low, but this system was significantly less toxic and more effective than Lipofectamine ${ }^{\circledR}$ or Nucleofection protocols which led to less than $1 \%$ HDR efficiency. In vivo evaluation of HDR effect has the been carried out on a model of DMD caused by mutations in the dystrophin gene. Interestingly, in vivo, CRISPR-AuNPs led, after an intramuscular injection, to an efficiency of $5.4 \%$ of the dystrophin gene correction in mdx mice (18 times higher than non-vectorised Cas9-RNP and donor DNA). This translated to improved mice muscle function for CRISPR-AuNPs treated mice. Therefore, this work established a proof of concept that non-viral delivery vehicles can generate HDR in vivo and have great potential for treating genetic diseases.

Still with the goal to vectorize genome editing tools, Ju et al. [43] recently reported a $\mathrm{pH}$-sensitive self-assembly of gold nanoclusters (AuNCs) with Streptococcus pyogenes Cas9 protein (SpCas9-AuNCs) (Figure 3E; Figure 5). AuNCs were first functionalised with GSH, and AuNC-GSH (negatively charged at physiological $\mathrm{pH}$ ) were then mixed with SpCas9 (positively charged) to form assembly driven by electrostatic forces. At acidic $\mathrm{pH}$, the carboxylic groups of AuNCs could reverse to partially protonated form, which would allow 
disassembly of the complexes and endosome disruption by proton sponge effect. SpCas9-AuNCs nanoparticles indeed successfully delivered spCas9 and efficiently knocked out the E6 oncogene in vitro, thus reestablishing the function of tumor-suppressive protein p53 and triggering apoptosis in cervical cancer cells while little effect was detected on ordinary human cells. Nevertheless, despite its promise, this system did not allow, for now, the co-delivery of sgRNA and therefore required a co-transfection restricting this system to in vitro use.

In parallel to their use for gene editing machinery, Au NP conjugated with an anti-His DNA aptamer (AuNP-His-Apt) were synthesized for selective binding of anticancerous Histagged proteins [44] (Figure 3D). This strategy was used to allow the transfer of the $11^{\text {th }}$ proapoptotic member of the BCL-2 (B-cell lymphoma 2) protein family, commonly called BIM. Interestingly, His-BIM delivered by AuNP-His-Apt was largely associated to mitochondria, in agreement with its biological function as an apoptosis triggering protein, and led to decreased viability of HeLa cells in a concentration-dependent manner. HeLa cell-derived cervical xenograft tumours co-injected with AuNP-His-Apt-His-BIM showed significantly reduced growth compared to tumours that received the nonsense control or protein alone. Systemic delivery was then evaluated into mice bearing EGFR-overexpressing A431 human epidermoid carcinoma cells xenografted tumours. To evaluate targeting ability, AuNP were loaded with His-BIM only or with both His-BIM (Alexa488) and GST-EGF (Texas Red). Interestingly, $12 \mathrm{~h}$ after IV administration, tumours exhibited a higher green signal when AuNP were loaded with both Alexa 488-labeled BIM and Texas Red-loaded EGF compared to BIM alone. Other organs (brain, liver, spleen, ovaries) and non-EGFR tumours did not show BIM accumulation. In a follow-up study, this system was evidenced to efficiently deliver recombinant proteins into mammalian cells (HeLa and 293T cell lines as well as cervical squamous carcinoma primary cells and J1 mouse embryonic stem cells) independently of their size, isoelectric point, and cellular localisation, proving the versatility of this platform [45], among the most deeply evaluated for protein in vivo delivery.

In the last five years, a set of AuNP have been investigated for their potential to deliver protein intracellularly, gene editing tools especially. Nevertheless, as already evocated, despite promising results, the long-term behaviour of Au NP still raises questions. It is now well established that after IV administration, Au NP are mainly found in elimination organs such as liver and spleen, as they are taken up by macrophages and accumulate in lysosome $[46,47]$. A recent study reveals an unexpected biotransformation arising between 2 and 6 months of Au NP uptake into human skin primary fibroblasts [48]. This phenomenon 
involved NP degradation followed by the formation, in a highly reproducible fashion, of nanostructures of $2.5 \mathrm{~nm}$ crystalline particles self-assembled into nanoleaves, still present in cell lysosome 6 months after AuNP exposition. This interesting study provides new evidence that $\mathrm{Au}$ NPs remain present in treated cells over a long period of time. Nevertheless, what would happen if the treatment envisaged required multiple injections? What would be the impact on treated cells in terms of toxicity? The clinical application of Au NP for protein delivery, and/or other drugs, will only be possible once these questions have been answered.

\subsection{Organic-inorganic hybrids}

Metal-Organic Frameworks (MOFs) are a class of hybrid materials composed of inorganic building units, metal ions, or metal oxide clusters, which are coordinative connected by organic linkers to create porous three-dimensional structures [49]. These crystalline materials are assembled by polyfunctional molecular building blocks and metal or metal cluster connecting nodes. Relying on high drug loading ability, various topologies and tunable pore size or surface chemistry, MOF represent a very attractive platform for drug delivery and have generated several convincing results especially for small molecules [50].

In 2012, Deng et al. established the possibility to expand MOF pore aperture toward sizes superior to $3.2 \mathrm{~nm}$, allowing loading of model proteins such as GFP [51]. The use of MOFs to protect proteins was then established by Liang et al. who associated MOFs with a wide range of proteins (including enzymes) using a biomimetic mineralization process avoiding organic solvent use [52] (Table 2). The proof of concept of intracellular delivery with MOFs was then first established by Roder et al. [53] who used oligohistidine-tagged protein and peptide to interact with coordinately unsaturated metal sites present at MOF surface. Three different MOF structures based on different metal components were studied: MIL-88A (Fe(III)-fumaric acid), HKUST-1 (Cu(II)-benzene-1,3,5-tricarboxylate), and Zrfum (Zirconium-fumaric acid). Zr-fum MOF was established as the most efficient to be internalised into HeLa cells. To evaluate the biological activity of complexed proteins, HisCytochrome C (His-CytC) was associated to $\mathrm{Zr}$-fum MOF and cell death was evaluated as a read out of cytoplasmic delivery and functionality into HeLa cells. Results showed a $40 \%$ cell death induced by His-CytC MOF treatment whereas this was not observed for MOF or His-CytC alone. This first study evidenced the interest of MOF for intracellular protein delivery in vitro, as the exposition of proteinoutside the structure could be poorly adapted for in vivo use. 
With the specific care to protect two proteins in a single device, antioxidative enzymes super oxide dismutase (SOD, $17 \mathrm{kDa}$ ) and catalase (CAT, $60 \mathrm{kDa}$ ) were loaded in nanoscale PCN-333 (Al) (NPCN-333) MOFs [54]. PCN-333 is a super-tetrahedron, composed of an aluminium trimeric cluster at the four vertices and triaminotrinitrobenzene ligands on the faces, in a vertex-sharing manner exposing 4.0 and $5.5 \mathrm{~nm}$ cavities of NPCN-333. This porosity allowed entrapment of both SOD $(2.8 \times 3.5 \times 4.2 \mathrm{~nm})$ and CAT $(4.9 \times 4.4 \times 5.6 \mathrm{~nm})$ in the same structure with loading efficiencies of 0.80 and $1.26 \mathrm{~g} . \mathrm{g}^{-1}$, respectively. Thereby obtained systems, associating both SOD (S) and CAT (C), were called SC@NPCN-333. After cell internalisation, this system allows SOD and CAT to mediate ROS intracellular detoxification, as assessed by protective effect when cells were treated with a redox-active compound. Indeed, when cells were treated with SC@FNPCN-333, more than $85 \%$ of cells survived even 7 days after treatment. Even if the biocompatibility of MOFs, in vitro and in vivo, needs to be further investigated, these results highlighted the potential of MOFs as "MOF-enzyme nanofactories" for protection and intracellular delivery of enzymes over extended periods.

The previously evocated process of MOF formation [52], relying on biomimetic mineralization to protect proteins and allow their cellular delivery was used by Chen et al. [55]. Low-cytotoxicity and biodegradable MOF materials $\left(\mathrm{Zn}^{2+}-2-\right.$ methylimidazole, ZIF-8) were stabilised with biocompatible polyvinylpyrrolidone (PVP) coating. By using protein as a starting element for MOF construction, this formulation method was used to entrap different proteins: BSA, EGFP, Red Fluorescent Protein (RFP), $\beta$-gal, caspase 3/BSA, BSA/RFP/ $\beta$-gal. All protein@ZIF-8 NPs were monodisperse, with a protein-dependent size (ranging from 87 nm for EGFP@ZIF-8 to 274 nm for BSA/RFP/ $\beta$-gal@ZIF-8). Interestingly, $\beta$-gal was shown to be protected from trypsin and $\alpha$-chymostrypsin mixture activity when loaded into ZIF- 8 NPs. Storage of $\beta$-gal was also considerably improved after three months at $4^{\circ} \mathrm{C}$, as $82 \%$ of free protein activity was lost versus $16 \%$ for ZIF-8-encapsulated protein. PVP-coated ZIF-8 NPs encapsulating FITC-labelled BSA exposed rapid internalisation in different cell lines (HeLa, MCF7, HaCaT, SKOV3, HepG2), with a substantial improvement compared to TAT peptide vectorisation of the same proteins in all evaluated cells. Finally, intracellular activity preservation of several delivered proteins ( $\beta$-gal, cas3, FITC-labelled anti tubulin antibody) was evidenced, establishing protein-entrapping biomineralized MOF NPs as an interesting platform for protein delivery and protein-based theranostics.

In parallel to PVP, coating of extracellular vesicles (EVs) derived from MDA-MB-231 tumour cells was used to improve stability and biocompatibility of ZIF-8 MOF loaded with BSA (60 kDa) or gelonin (28 kDa) [56]. This coating was evidenced in vitro to reduce particle 
opsonisation (by nearly 6-fold compared to non-coated MOF) and macrophage uptake. Such coated MOF loaded with FITC BSA favored tumour cell internalisation specifically toward MDA-MB-231 cells with $77.5 \%$ for coated MOF vs. $43.7 \%$ for MOF or less than $12 \%$ for free FITC-BSA). MOF nanoparticles loaded with gelonin, a $N$-glycosidase protein triggering cell apoptosis by cutting a specific glycosidic bond in rRNA, induced significant cell apoptosis. This has been demonstrated in vivo in orthotopic MDA-MB-231 tumour-bearing mice, coated and uncoated MOF NPs loaded with fluorescent gelonin, which were shown to accumulate about 11- and 4-fold more respectively than protein alone 3 days after IV injection. This improvement in tumour accumulation translated to a significant decrease in tumour growth when mice were treated with coated MOF NPs, evidencing their interest for in vivo intracellular delivery after systemic administration, even if the long-term safety of MOFs in vivo still needs to be ascertained.

\subsection{Carbon-based nanoparticles}

\subsubsection{Carbon nanotubes}

In the beginning of the 2000's, Dai's group initiated the use of carbon nanotubes for intracellular protein delivery, by establishing the proof of concept of delivery of various proteins (streptavidin, protein $\mathrm{A}, \mathrm{BSA}$, and $\mathrm{CytC}$ ) via non-covalent and non-specific binding to nanotube sidewalls into several cell lines: HeLa, NIH3T3, Jurkat, and HL60 [57, 58]. Hydrophobic unoxidized areas of the nanotubes were reported to induce interactions with cell membrane and a subsequent endocytosis. Through the apoptotic activity induced by delivered CytC, while non loaded SWNT treatment induce no significant cytotoxicity, they established the ability to deliver a functionally active protein into cell cytoplasm. Recently, Li et al conjugated single-walled carbon nanotubes (SWCNTs) to a protein of interest via a streptavidin-desthiobiotin (SA-DTB) linkage, sensitive to near infra-red (NIR) light stimulation to control protein release in the cytoplasm [59]. Saporin (Sap), a ribosome inactivating protein, inhibiting protein translation and inducing apoptosis, was conjugated through such SA-DTB linkage to SWCNTs. Thereby formed SWCNT-SA-DTB-Sap were delivered intracellularly where they induced efficient cell death upon NIR irradiation. After intravenous injection into mice bearing subcutaneous HeLa tumour xenografts, SWCNT-SADTB-nEGFP were shown to accumulate in the tumour by enhanced permeability and retention effect (EPR). After 4h, NIR stimulation induced an important release and nuclear translocation of nEGFP was detected compared to non-NIR irradiated tumours. Nevertheless, 
in regards with thrombus formation arising after intravenous intravenous injection of carbon nanotubes [60], this system could only be dedicated to intratumoral administration.

\subsubsection{Carbon dots}

Among carbon nanomaterials, carbon dots (CDs) are of particular interest for therapeutic applications due to their elevated water solubility, high photostability, easy synthesis as well as their natural cell permeability properties. CDs were used to complex and deliver EGFP as a model protein [61]. In vitro, HeLa cells treated with CD-EGFP exhibited significantly stronger green fluorescence compared to treatment with free EGFP, evidencing the important role of CDs in transferring proteins into cells. Unfortunately, no proof of functional protein delivery has been provided to date.

\section{Organic nanocarriers}

\subsection{Polymer-based nanocarriers}

Various polymeric delivery systems have been considered for intracellular protein delivery and achieved much progress. According to the used polymers, the preparation processes, different structures were found (i) core-shell nanocapsules (NCs), matrix based nanospheres (NS), or auto assembled systems (micelles) as well as dendrimers (Figure 4; Table 3). On the following section, we decided to describe the more recent systems based on these different structures.

\subsubsection{Polymer nanocapsules}

\subsubsection{Crosslinked polymer nanocapsules}

Li et al. [62] have developed a polymeric vector based on protein NCs (nProteins), which were formed by in situ growth of thin layers of polymer (shells) around single protein molecules (cores). Poly-(allylamine hydrochloride) (PAH) was alternatively associated to poly(ethylene glycol) (PEG) and thiol moieties. Indeed, PEG moieties were used to minimize the cytotoxicity commonly associated with cationic polymeric vectors. Negatively charged proteins therefore spontaneously assembled, by electrostatic interactions, with positively charged modified PAH. Then, oxidation of the thiol functions on the modified PAH formed crosslinked nProteins. Upon internalisation of the nProteins through endocytosis, their disulphide bonds could be cleaved by glutathione (GSH) within cytosol, releasing the protein cargos. nBSA particles were formed $(20-25 \mathrm{~nm},+14 \mathrm{mV})$ and showed an internalisation into HeLa cells 100 times more important than native BSA. Caspase-3 (Cas3) was then employed as a model protein for its capacity to induce an apoptosis signal. nCas 3 treated cells exposed 
high apoptosis levels compared to untreated cells or even cell treated with free Cas3 (77\% of annexin V positive cells for nCas3 vs. $1.87 \%$ for non treated cells and $1.85 \%$ for Cas 3 treated cells). This result has confirmed that the crosslinking structures in the protein-polymer nanocapsules were probably cleaved by GSH, allowing the release of fully functional protein after cell internalisation. Nevertheless, these nanocapsules are restricted to the encapsulation of negatively charged proteins.

\subsubsection{Acrylamides based polymeric nanocapsules}

In 2009, the Yi Tang's group developed a smart method to vectorize functional enzymes in cell cytoplasm [63]. To allow synthesis of single-protein nanocapsules (NCs), mature caspase 3 (Cas3) was mixed with positively charged acrylamide (AAm) monomers, N(3-Aminopropryl) methacrylamide (APMAAm) monomers and bis-acrolylated short peptide crosslinkers that can be specifically cleaved by Cas3 protease. After physical adsorption of the monomers as well as crosslinkers onto the protein surface, in situ free-radical polymerization was achieved to form $13 \mathrm{~nm}$ NCs. Interestingly, when incubated at $37^{\circ} \mathrm{C}$, NCs were cleaved by the caspase activity of the encapsulated protein itself. In vitro, cells treated with Cas3 NCs led to an increased apoptosis, exposing extensive apoptotic DNA fragmentation as well as decrease cell proliferation in different treated cells lines (HeLa, MCF7, M249). To obtain spatiotemporal control of NCs degradation, the same peptide crosslinker was modified with a photolabile o-nitrobenzyl ester motif. In this new configuration, only synergic action of UV radiation and Cas3 hydrolysis led to cells apoptosis [63]. After this proof of concept on self-degrading NCs, the protease-modulated design was convert to be applied to degradation by external proteases. The same monomer couple was used, but a ubiquitous endoprotease (furin) cleavable peptide was used as crosslinker to allow delivery of a wider range of proteins [64]. By using $\mathrm{CHO}$ cell lines modified to express varying furin concentrations (low, normal high), they evidenced that the presence of active intracellular furin and furin-degradable NCs were both required for successful eGFP-Nuclear Localisation Signal (NLS) nuclear delivery. The authors established the versatility of their NC platform to deliver protein cargos of different sizes and tertiary structures (caspase 3, transcription factor KLf4) without loss of bioactivity. The same NC were synthesized using a different degradation strategy relying on the use of a redox-responsive, disulfide-containing crosslinker (N,N'- bis(acryloyl) cystamine) [65]. The polymer shell underwent degradation and cargo release into cytosol reducing conditions while this was not observed in nonreductive conditions. This platform also proved its efficiency for protein intracellular delivery. 
These two type of NC (furin or redox sensitive NC) were then PEGylated and exhibited a similar ability to deliver in vitro a recombinant myogenic transcription factor (MyoD) into myoblast nucleus leading to the induction of effective myogenic differentiation [66]. The redox-sensitive NCs encapsulating recombinant p53 were then modified via a copper free click reaction allowing the attachment of ethylene glycol motifs which could also be conjugated to a targeting ligand, the luteinizing hormone releasing hormone (LHRH) peptide [67]. LHRH functionalised p53-NCs were shown to selectively deliver recombinant p53 into both the cytoplasm and nuclei of MDA-MB-231 cells expressing LHRH receptor leading to an apoptosis signal $\left(\mathrm{IC}_{50} \sim 300 \mathrm{nM}\right.$ ). Unfortunately, to our knowledge, these different systems have not been evaluated in vivo yet; their small size (15-30nm) would make them particularly relevant while considering IV administration.

Also relying on stimuli responsive system, Liu et al. [68] recently proposed a tumourrelated stimuli activated nanogel (aNG) by nano-in-nano hierarchical assembly of a core-shell polymeric nanogel loaded with previously described redox responsive nanocapsules [67] to encapsulate RNase A. Tetracycline antibiotic Doxycycline (Doc), was reported to eradicate cancerous stem cells (CSCs) by interacting with mitochondria in breast cancer [69]. This chemical was thus selected to be co-encapsulated in aNG with the cytotoxic protein RNase A. Doc and R-rNC co-loaded nanogel (Doc/R-rNC/aNG) were loaded in a polymeric nanogel core formed by polymerization of three monomers, AAm, APMAAm, and a synthetic azidedecorated monomer (AAm-N3). The $\mathrm{N}_{3}$ function associated to the APMAAm monomer enabled the conjugation with DCBO-hyaluronic acid (HA) by copper free click chemistry permitting the formation of a shell allowing a high binding affinity to the CD44 receptors over-expressed on both CSCs and cancer cells [70]. The final system (Doc/R-rNC/aNG) were $100 \mathrm{~nm}$ in hydrodynamic diameter and expose a slightly negative zeta potential. In vitro, Doc/R-rNC/aNG exposed a cytotoxic effect toward mammosphere cells significantly higher than that of R-rNC alone ( $\mathrm{IC}_{50}$ of $65 \mathrm{nM}$ vs. $366 \mathrm{nM}$, respectively). Combinational antitumour efficacy of Doc/R-rNC/aNG was then evaluated in vivo on a MDA-MB-231 xenograft tumor mouse model. After intravenous injection of Doc/R-rNC/aNG, an inhibitory ratio of approximately $73.6 \%$ compared to tumour weight of PBS treated mice was evidenced. This system therefore provides a new promising strategy for the consecutive delivery of multiple therapeutics for targeting both CSCs and classic tumour cells.

\subsubsection{Polymersomes}


Chimeric polymersomes (CP) functionalised with apolipoprotein E peptides (ApoE) were used for glioblastoma (GBM) targeting [71]. Indeed, ApoE has a high affinity for multiple low-density lipoprotein (LDL) receptor families highly expressed at the blood-brain barrier (BBB). CP were synthesized from poly (ethylene glycol)-b-poly (dithiolanetrimethylenecarbonate-co-trimethylenecarbonate)-b-polyethylenimine triblock copolymer [PEG-P(DTC-TMC)-PEI] and ApoE peptide-modified PEG-P(DTC-TMC) diblock copolymer [ApoE-PEG-P(DTC-TMC)]. PEI was reported to be mainly positioned on the inner side, therefore enabling protein complexation and protection into polymersome lumen. The polymersomal membrane consisting of P(DTC-TMC) was self-crosslinked during formulation process giving stiff vesicles able to disassemble under cytoplasmic reductive condition allowing protein release [71]. ApoE-CP loaded with cytotoxic protein saporin (Sap) were prepared by simple addition of Sap to a copolymer solution. Protein association efficiency, driven by electrostatic interaction was evaluated at 74-92\% (w/w). ApoE-CP-Sap particles were $80-86 \mathrm{~nm}$ in diameter, exposed a vesicular morphology and a neutral zeta potential. After receptor-mediated endocytosis in U87 cell lines, ApoE-CP-Sap were able to induce a cytotoxic effect through upregulation of ErkA/2, Bax pathway. ${ }^{125}$ I-labeled Sap, used to study pharmacokinetics, showed that ApoE-CP-Sap intravenously injected in mice had a longer elimination half-life $\left(\mathrm{t}_{1 / 2 \beta} \approx 5 \mathrm{~h}\right)$ compared to free Sap $\left(\mathrm{t}_{1 / 2 \beta} \approx 1.1 \mathrm{~h}\right)$ and were able to accumulate in tumoral tissues and induced apoptosis. Interestingly, histological analyses revealed no organ damage during ApoE-CP- or CP-SAP treatment. Mice median survival time following ApoE-CP-Sap was 59 days whereas no mice treated with free Sap or PBS survived past 24 days. Survival benefit was increase compared to chemotherapeutics-based nanomedicines recently described for orthotopic GBM bearing mice (median survival time: 28-37 days) [72, 73]. This is likely due to ApoE-mediated efficient blood-brain barrier penetration and targeted GBM cell uptake, as well as to high potency and low adverse effects of protein vs. synthetic molecules.

\subsubsection{Polymer nanospheres}

\subsubsection{Polyester based nanospheres}

Using a double emulsion solvent evaporation method, Singhal et al. [74], developed catalase-loaded poly(lactic co-glycolic acid) (PLGA) nanoparticles (NPs) to protect human neurons against oxidative damage. Indeed catalase, an $\mathrm{H}_{2} \mathrm{O}_{2}$-degrading enzyme, is an 
important antioxidant. The average hydrodynamic diameter of catalase-loaded NPs (NanoCAT) was $280 \mathrm{~nm}$ with a zeta potential of $+20 \mathrm{mV}$. The encapsulation efficiency of catalase was $99 \%(\mathrm{w} / \mathrm{w})$. In vitro, Nano-CAT $(50-200 \mu \mathrm{g} / \mathrm{ml})$ conserved cell viability in a dosedependent manner in primary human neurons treated with $\mathrm{H}_{2} \mathrm{O}_{2}$. Nano-CAT-mediated neuroprotection was also evidenced following $24 \mathrm{~h}$ of $\mathrm{H}_{2} \mathrm{O}_{2}$ treatment. Nano-CAT significantly decrease protein oxidation level, evidencing an effective CAT delivery and preservation from direct oxidation of proteins. Altogether, these in vitro results were promising for neuron protection. Unfortunately, to our knowledge, no in vivo evaluation has been performed yet.

Self assembled nanoparticles were developed by Rui et al. [75] who recently synthesized a series of hyperbranched cationic poly( $\beta$-amino ester)s (PBAEs) containing both cationic and anionic charges for the encapsulation of a broad variety of proteins (from 27 to $160 \mathrm{kDa}$ ). NPs formation occurs by simple mixing of polymer and proteins solubilised in aqueous buffer. A carboxylate ligand was used to increase polymer-protein interactions via hydrophobic interaction in parrallel to electrostatic interactions. Polymers terminated with a carboxylate ligand of moderate hydrophobicity (C5), were the most appropriate for cellular internalisation and endosomal disruption. C5 polymers allowed functional delivery of Cas9RNP (160 kDa) to trigger CRISPR gene editing. In vitro, delivery of Cas9-RNPs directed toward GFP as a reporter gene resulted in nearly $80 \%$ GFP knockout. In regard to commercial reagents reagents (Lipofectamine ${ }^{\circledR}$, CRISPRMax and jetCRISPR), C5/Cas9-RNP nanoparticles allowed significantly higher levers of gene editing (16\% and 43\% in GL261 and B16 cells, respectively). Furthermore, with the same nanoparticles, in vitro co-delivery of Cas9-RNPs targeting the human CXCR4 gene and a donor DNA repair template enabled $4 \%$ homology-directed repair (HDR) in HEK-293T. Furthermore, C5/Cas9-RNP NPs were in vivo evaluated in a mouse glioma model where it induced CRISPR gene editing upon intracranial injection. This polymer strategy is among the most promising developed to date in the polymer field.

\subsubsection{Nanogels}

pH-sensitive nanogels were developped by Zhang et al. [76] by associating a boronate ester cross-linked zwitterionic nanogel (NGCA) with ATP/pH-sensitivity to reach a two-stage charge conversion responding to $\mathrm{pH}$ found in extracellular media $(\mathrm{pH}$ 6.5-6.8) and to endosomal compartments ( $\mathrm{pH}$ 5-6). NGCA were formed thanks to interactions between positively charged amino groups (dendritic polyglycerol (dPG)) and negatively charged 
carboxyl groups (2-formylphenylboronic acid (FPBA) with different citraconic amide (CA) functional percentages). Thu formed nanogels are supposed to become positive once accumulated into slightly acidic tumour tissue to favor endocytosis while hydrolysis of CA in the acidic endo/lysosome would lead to exposure of more amine functions able to trigger endosomal escape by proton sponge effect. The high concentration of cytoplasmic ATP is then supposed to induce nanogel degradation and subsequent protein release as ATP can break the boronate ester cross-linkers of the nanogel scaffold by competing with dPG. The encapsulation of CytC (12 kDa), positively charged at physiological $\mathrm{pH}$, was achieved by its co-precipitation with the macromolecular precursors (anionic carboxyl groups). Nanogels were stabilized via electrostatic interactions with anionic carboxyl groups. The loading capacity of CytC reached $21 \%$ for NG-50\%CA. In vitro release study confirmed CytC release increased at $\mathrm{pH}$ 6.5, and in intracellular ATP environment (5 mM ATP solution at $\mathrm{pH} 7.4$ ) compared to $\mathrm{pH} 7.4$ media. Biological activity of $\mathrm{CytC}$, the ability to induce a mitochondria dependent apoptosis, was then evidenced into MCF-7 cells with NGCA (27.8\% of apoptosis induction). Such nanogel carriers therefore seem to enhance the transfer of positively charged proteins into cytoplasm.

Hybrid nanogels inspired by molecular chaperones were designed by Kawasaki et al. to allow cytoplasmic protein delivery using an external magnetic field [77]. Hybrid magnetic nanogel chaperons (MC) were therefore synthesized by mixing an aqueous nanogel suspension formed with cholesterol-bearing pullulan and hydrophobized iron oxide NP suspended in tetrahydrofuran. To allow proteins loading (BSA, insulin and $\beta$-gal), a simple co-incubation with iron oxide NP was performed. MC exposed sizes comprised between 100 $\mathrm{nm}$ and $180 \mathrm{~nm}$ (with BSA), and a neutral zeta-potential. A prodrug assay was conducted using $\beta$-gal conjugated with cytotoxic 5-fluorouridine (5-fluorouridine-5'-O- $\beta$ galactopyranoside (5-FUR- $\beta$-gal) as a model prodrug substrate. The $\beta$-gal delivered by MC in Hela cells showed enzymatic activity within cells and converted non-cytotoxic 5-FUR- $\beta$-gal into cytotoxic 5-FUR. However, this system, despite its smart design, is probably restricted to an in vitro use, as the authors reported that protein release was obtained when adding of foetal bovine serum to the complex, a practice poorly compatible with an in vivo use.

Receptor targeted nanogels were synthesized by Zhiyuan Zhong's group to deliver cytotoxic proteins (CytC \& granzyme B $(\mathrm{GrB})$ ) to human cancer cells SKOV3 (ovarian) and MDA-MB-231 (breast), overexpressing EGFR and CD44 receptors [78]. Hyaluronic acid (HA) was chosen for its natural interaction with CD44 and it was linked with GE11 (YHWYGYTPQNVI) peptide able to target epidermal growth factor receptor (EGFR). 
Redox-sensitive EGFR/CD44-NGS were obtained by nanoprecipitation and subsequent photo click crosslinking thanks to the grafting of tetrazol and cystamine methacrylamide to HA allowing a tetrazole-alkene photo-click chemistry. The cystamine moieties bring nanogels reduction-sensitivity, triggering the release of encapsulated protein under high GSH cytoplasmic concentration. CytC was efficiently loaded with over $99 \%$ (w/w) efficiency. CytC-NGs expose a diameter size of 164-168 nm and a slightly negative zeta potential (-15 $\mathrm{mV}$ ). After internalisation of NGs, the protein was shown to be delivered into the cytoplasm of SKOV3 cells, suggesting endosomal escape. Intravenously injected GrB-EGFR/CD44-NGs strongly inhibited tumour growth in SKOV3 and MDA-MB-231 mice tumor models, with rates of $87 \%$ and $82 \%$ respectively and negligible systemic toxicity. These effects were achieved with low doses of $3.85 \mathrm{nmol}$ of GrB equivalent $/ \mathrm{kg}$ (equiv./kg), which is more than 1000 times lower than needed with doxorubicin and platinum-loaded nanomedicines [79, 80]. With the goal of treating metastatic breast cancer, saporin (Sap) was encapsulated in the same formulations forming Sap-EGFR/CD44-NGs [81]. In 4T1-luc metastatic mice breast cancer model, Sap-EGFR/CD44-NGs exposed significant inhibition of tumour metastasis to lungs at a low dose (3.33 nmol Sap equiv./kg). Using higher dosage (13.3 nmol Sap equiv./kg) resulted in additional reduced lung metastasis without detected adverse effects. Still using the same formulation process, Huang et al. [82] developed HA-NG grafted with cationic lysine and the endosome disruptive GALA peptide. NGs were synthesized from HA-g-cystaminemethacrylate (HA-g-Cys-MA) and HA-g-lysine-tetrazole/GALA (HA-g-Tet/GALA), using, instead of previously described nanoprecipitation, a microfluidics technique to associate different proteins association including Sap. SAP-NG/GALA treatment led to enhanced anticancer effect with smaller IC $_{50}$ values compared to NGs without GALA suggesting that such endosome disruptive peptide facilitates the fast endosomal escape of protein-loaded NGs. After IV injection, ${ }^{125}$ I-Sap-NG displayed an increased stability in blood (half-life $\mathrm{t}_{1 / 2}=$ $3.80 \mathrm{~h})$, by contrast to free Sap which was rapidly cleared from the systemic circulation $\left(\mathrm{t}_{1 / 2}=\right.$ $0.69 \mathrm{~h})$. After IV injection in mice bearing $4 \mathrm{~T} 1$ tumours, ${ }^{125} \mathrm{I}-\mathrm{Sap}-\mathrm{NG}$ exhibited increased ( 4 fold) protein accumulation at tumour sites at $6 \mathrm{~h}$ compared to free ${ }^{125} \mathrm{I}-\mathrm{Sap}$. Thus, the Sap loaded NGs possesses convincing tumour targetability and extended circulation time. Interestingly, although exhibiting a shorter elimination half-life, smaller NGs displayed higher tumour accumulation. 


\subsubsection{Micelles}

Polyionic complex PIC micelles composed of PEG-poly[N-[N'-(2-aminoethyl)-2aminoethyl]aspartamide] (PEG-pAsp(DET)) were developed for protein cytoplasmic delivery by Kataoka's group, pioneer in protein loading with a charge conversion step [83]. To enhance electrostatic interactions with Asp cationic part, a reversible modification was performed on protein lysine groups which were modified with citraconic amides (cit) or cisaconitic amides (cis), thus decreasing the protein isoelectric point (pI) and increasing anionic charge density. PIC micelles were expected to release the original protein once inside endosomes, as the acidic environment would cause charge conversion. Encapsulation of different proteins types was assessed and the behavior of loaded micelles in vitro investigated. CytC-loaded PIC (CytC@PIC) micelles were synthesized and turned out to be stable at pH 7.4 , monodisperse, $50 \mathrm{~nm}$ in diameter while in vitro dissociation was evidenced at $\mathrm{pH} 5.5$ allowing the release of functional CytC. Alexa488-CytC@PIC micelles were efficiently delivered to human hepatoma HuH-7 cells and escaped endolysosomes, whereas unencapsulated protein was not. Similar PIC micelles were used to efficiently deliver functional anti-nuclear pore complex (NPC) IgG into into living cells, highlighting that antibody charge conversions did not disturb its ability to interact with its intracellular target [84]. The degree of Cis Aco modifications on IgG lysins, as well as the presence of homocatiomer PAsp(DET) into PEG-PAsp(DET)-based PIC micelles were then optimised to increase PIC micelles stability [85]. Such PIC micelles, associated with anti-NPC IgG modified with $50 \%$ citconversion and $75 \%$ PAsp(DET), exhibited a significantly higher intracellular delivery of antibodies.

chimaeric lipopepsomes (CLP) were formulated by Qiu et al. [86] using asymmetric poly(ethylene glycol)- $b$-poly( $\alpha$-aminopalmitic acid)- $b$-poly(l-aspartic acid) (PEG- $b$-PAPA- $b$ PAsp) triblock copolymer functionalized with cyclic RGD peptide (cRGD-CLP) to interact with $\alpha_{v} \beta_{3}$ integrin. Using L-Arg moieties, saporin (Sap) was complexed to cRGD-CLP by electrostatic interactions to form Sap-cRGD-CLP characterised by a size of $66 \mathrm{~nm}$ and a surface charge of $-7.7 \mathrm{mV}$. Sap-cRGD-CLP exposed a significant antitumour effect toward A549 human lung cancer cells overexpressing $\alpha_{\mathrm{v}} \beta_{3}$ integrin ( $\mathrm{IC}_{50} 16.3 \mathrm{nM}$ ). This effect was not observed with free proteins. In vivo, antitumour activity was evaluated after IV administrations (4 injections every 4 days) of Sap-cRGD-CLP in A549-Luc orthotopically xenografted human lung tumours in mice. SAP-cRFG-CLP efficiently inhibited tumor growth 
and allowed increase in mice survival time (median survival time 50 vs 28 days for nontargeted control SAP-CLP).

\subsubsection{Dendrimers}

Dendrimers have been explored as intracellular protein delivery systems after various chemical modifications to favor interactions with positively or negatively charged proteins. Hence, polyamidoamine (PAMAM) dendrimers where modified with phenylboronic acid (PBA), that can interact with the guanidyl group of arginine present in proteins via guanidinium- $\pi$ interactions [87]. This modification indeed allowed loading of a broad range of proteins including fluorescent proteins BSA, R-PE, GFP, YFP, RFP, CytC, trypsin, lysozyme, $\beta$-gal, HRP, RNase A, Sap, trypsin, endonuclease Cas9 forming complexes of 100-300nm in size (Table 3) [88]. The use of PBA (vs. other boronic acid derivatives) as well as the number of PBA molecules conjugated with each dendrimer, were evidenced as a crucial parameter to increase delivery efficiency. Interestingly, dendrimers showed better efficiency to deliver functional proteins ( $\beta$-gal, HRP) compared to commercial reagents PULSin ${ }^{\circledR}$ (Polyplus transfection, France) and TransEx (Cenji Biotech, China) used as positive controls. RNase A, Sap and trypsin were found to be highly cytotoxic ( $70 \%$ cell death) when delivered by dendrimers while nontoxic when incubated alone with human breast cancer MDA-MB-231 cells. Finally, PBA dendrimers were used to deliver Cas9-RNP. GFP-targeting Cas9-RNP were delivered to HEK293 cells expressing GFP and reduced GFP expression (40\% of GFP inhibition), more potently than commercial reagent CRISPRMax. Cas9-RNP targeting AAVS1 and $H B B$ genome loci efficiently edited them in human colon cancer HCT-116 cells. Cas9RNP targeting CTNNB1 gene knocked out this gene. Overall, this system shows great promise for the intracellular delivery of a wide range of proteins, although it will have to be tested further in vivo.

Still to reinforce binding between cationic polymers and proteins, use of guanidiniumrich polymers constitutes another strategy. Indeed, guanidinium can form salt bridges and hydrogen bonds with amides and carboxyl groups in proteins [89, 90]. Chang et al. [91] used this strategy and synthesized polymers composed of three parts: (i) a dendrimer scaffold (amine-terminated generation 5 polyamidoamine), (ii) a multivalent protein binding surface (composed of guanidyl groups), (iii) a hydrophobic membrane-disruptive region (hydrophobic phenyl group). Polymers were designated as DGBA as they were dendrimers (D) displaying 4-guanidylbenzoicacid (GBA). The complexation of proteins with DGBA dendrimers was 
carried out by simple co-incubation. FITC-BSA-DGBA complexes $(240 \mathrm{~nm},+34 \mathrm{mV})$ were efficiently internalised into human cervical cancer HeLa cells and were localised diffusely in the cytoplasm after $2 \mathrm{~h}$. DGBA was much more efficient than the commercial protein delivery reagent PULSin ${ }^{\circledR}$ in delivering BSA-FITC into HeLa cells, while exposing negligible cytotoxicity. Large proteins as R-phycoerythrin (R-PE, $240 \mathrm{kDa}$ ) and $\beta$-gal tetramer (430 kDa) were delivered by DGBA dendrimers into HeLa, NIH3T3, and Raw264.7 cells. X-gal staining evidenced that delivered $\beta$-gal retained its activity. Finally, DGBA was used to deliver apoptosis triggering proteins (p53, Sap) to HeLa and human lung carcinoma PC-9 cells. DGBA-p53 activated p21 and induced apoptosis ( 25\%, annexin V-PI staining). DGBA-Sap inhibited cell proliferation ( $\mathrm{IC}_{50}: 2.2 \mathrm{nM}$ ), and inhibited tumour growth in PC-9 xenograft mice, with minimal systemic toxicity.

Polymer interest for intracellular protein delivery is therefore now clearly established, with some promising systems exposing convincing in vivo results. The next step will be their translation into the clinic, as to our knowledge, no clinical trial using polymer-based protein delivery systems is currently ongoing.

\subsection{Lipid based nanocarriers}

Liposomes technology has been evaluated for four decades for the delivery of drugs and is now fully mature and validated for the marketing of many specialty drugs (Caelyx ${ }^{\circledR}$, Daunosome $^{\circledR}$, Myocet $^{\circledR}$, Ambisome ${ }^{\circledR}$ ). Even if not necessarily for intracellular delivery, liposomes have also been used for protein delivery for decades, starting with reference articles published in the 1970s and 1980s, followed by numerous studies till this day [92, 93]. Proteins have been incorporated into liposomes by multiple processes such as reverse-phase evaporation, injection, freeze-thaw cycles, extrusion, dry lipid hydration, and leading to variable association efficiencies [92]. Nevertheless, to this day, no commercial liposomal protein delivery system has reached the market, especially for intracellular delivery. The research in this field is however very active, as underlined by the following studies describing different lipid NP compositions, mainly based on cationic lipids but also on neutral lipid or lipopolymers (Table 4, Figure 4).

\subsubsection{Cationic lipid based commercial reagents}


Commercial transfection reagent such as Lipofectamine ${ }^{\circledR}$ RNAiMAX and 2000 were evaluated for the intracellular delivery of negatively charged Cas9-RNPs [94]. Using Lipofectamine $^{\circledR}$ 2000, Cas9-RNPs and single-stranded DNA oligonucleotide (ssODN) donor template, in vitro homology directed repair (HDR) was demonstrated in an EGFP-repair reported line, and EGFP HDR frequencies of 8-11\% were achieved. Interestingly, when targeting therapeutically relevant EMX1, CLTA2 and VEGF genes with Cas9-RNPs delivered by RNAiMAX ${ }^{\circledR}$, all three genes were cleaved with efficiencies similar or greater (depending on targeted gene) than with plasmid transfection method and with less off-target effects. In vivo, RNAiMAX ${ }^{\circledR}$ was used to deliver Cas9-RNPs targeting GFP into postnatal day 2 (P2) transgenic Atoh1-GFP mouse cochlea which had only hair cells expressing GFP: $13 \%$ of cells lost GFP expression near the administration site, with minimal hair cell toxicity. In the same model, when using Lipofectamine ${ }^{\circledR} 2000,20 \%$ of cells lost GFP expression, and no toxicity was observed in those conditions. Altogether, this study suggests Lipofectamine ${ }^{\circledR}$ RNAiMAX or 2000 could be used to deliver Cas9-RNPs to treat genetic conditions causing deafness. Yu et al. [95] demonstrated the efficiency of novel liposomal transfection reagent Lipofectamine ${ }^{\circledR}$ CRISPRMAX to deliver Cas9-RNPs to mammalian cells. Such formulations were shown to generate more Indels (insertions/deletions) at the chosen HPRT1 locus than Lipofectamine $^{\circledR}$ RNAiMAX or 3000 in "easy-to-transfect" cell lines (HEK293, HeLa, and U2OS) as well as "hard-to-transfect" cell lines (HepG2, A549, and MCF-7). CRISPRMAX ${ }^{\circledR}$ resulted in 15 to $40 \%$ increase in genome cleavage efficiency compared to Lipofectamine ${ }^{\circledR}$ 3000 or RNAiMAX respectively. Co-delivery of donor DNA with Cas9-RNPs into GripTite HEK293 cells (disrupted EmGFP gene) was achieved using Lipofectamine CRISPRMAX. After $48 \mathrm{~h}$, up to $17 \%$ of cells had a restored EmGFP activity. However, it should be noted that compared to Lipofectamine ${ }^{\circledR}$ CRISPRMax, electroporation resulted in 94, 91 and $44 \%$ Indel production efficiencies in Jurkat T cells, K562 and SC-1 cells respectively.

Altogether, similarly to data obtained for nucleic acid delivery, cationic lipid-based commercial vectors could be an interesting option for ex-vivo modification (depending on cell type) or for local administration in vivo, IV injection being difficult in regards to the potential toxicity of these cationic lipids.

\subsubsection{Cationic lipid-based systems}

Various cationic lipid formulations were investigated to deliver proteins. Among the first developed, BioPorter ${ }^{\circledR}$ was used to complex a set of proteins (fluorescently tagged antibodies $(\mathrm{Ab})$, phycoerythrin, caspase 3 , caspase 8 , granzyme $\mathrm{B}$, and $\beta$-gal) and insure their 
cytoplasmic delivery, establishing references in the field [96]. BioPorter ${ }^{\circledR}$ is constituted by a trifluoroacetylated lipopolyamine (TFA-DODAPL (2,6-diamino-hexanoic acid [5-amino-5[5-[2-amino-6-(2,6-diaminohexanoylamino)-hexanoylamino]-1-(3-dioctadecylaminopropylcarbamoyl) pentylcarbamoyl] - pentyl] - amide)) cationic lipid associated with DOPE (1,2-dioleoyl-sn-glycero-3-phosphoethanolamine) to form liposomes. Interestingly, this formulation allowed protein delivery in $70-75 \%$ of NIH3T3 cells by contrast to other evaluated formulations (DOTAP:DOPE, DMRIE (1,2-dimyristyloxy-propyl-3-dimethylhydroxy ethyl ammonium bromide), DOPG (1,2-Dioleoyl-sn-glycero-3-phosphoglycerol), DOPC (1,2-Dioleoyl-sn-Glycero-3-Phosphocholine), Trans-IT ${ }^{\circledR}$, FuGene $^{\circledR}$ 6, Tranfast $^{\circledR}$, Lipofectamine $^{\circledR}$ and Lipofectin ${ }^{\circledR}$ ) leading to less than 5\% delivery efficiency of FITC-Ab or $\beta$-gal. The versatility of this delivery was evidenced in various cell lines including HEK293, MDCK, P19. In Jurkat cells, TFA-DODAPL: DOPE/granzyme B led to increased apoptosis (30\% of cells were annexin-V positive compared to $3.6 \%$ for non treated cells or $4.1 \%$ for TFA-DODAPL:DOPE alone). Nevertheless, CytC, positively charged at physiological pH similarly to granzymeB, complexed with TFA-DODAPL failed to induce apoptosis in the same cell type, evidencing the crucial role of hydrophobic interactions, as well as conformation of the protein to be efficiently delivered by TFA-DODAPL. BioPorter ${ }^{\circledR}$ system was also used to allow efficient cytoplasmic and nuclear delivery of a protein nucleoside kinase to allow gene suicide therapy [97]. This delivery strategy was shown to increase sensitivity to cytotoxic nucleoside analogues, such as Ganciclovir ${ }^{\mathrm{TM}}$, into human osteosarcoma and CHO cell lines. To our knowledge, no in vivo evaluation of BioPorter ${ }^{\circledR}$ has been performed to date.

Synthetic systems initially developed for nucleic acid delivery were then evaluated for protein delivery. In this context, Behr's group used dioctadecylglycylspermine (DOGS) to intracellularly vectorize a panel of negatively charged proteins (FITC-IgG-aactin, FITC-IgGatubulin, R-Phycoerythrin, BSA) [98]. DOGS/BSA mix formed large complexes (500-900 $\mathrm{nm}$ ) and charge ratios (M:M) from 32 to 1100 were necessary to obtain BSA delivery (by contrast to a charge ratio of 6 for nucleic acids). Interestingly, depending on the complexed protein, the intracellular distribution of DOGS-protein complex was different, as shown in three cell lines (Baby hamster kidney (BHK), Chinese hamster ovary (CHO) or immortalized line of human $\mathrm{T}$ lymphocyte Jurkat cell lines). Indeed, BSA localisation was mainly punctiform, evidencing a lack of endosomal escape probably due hydrophobic protein-lipid interactions interfering with complex dissociation. By contrast, FITC-IgG-DOGS treatment led to diffuse cytoplasmic delivery. This study therefore also confirmed the role of attractive 
Van der Waals interactions, in parallel to electrostatic ones, to influence intracellular delivery of proteins. Indeed, the protein surface area, representing the number of lipid molecules per square nanometre of protein surface, was highlighted as the most important parameter to drive the delivery characteristics. These systems, relying on large complex with a tendency to aggregate, seem however to be restricted to an in vitro use. Complexes composed of SAINT-2 cationic lipids ( $N$-methyl-4(dioleyl)methyl-pyridinium-chloride) and lipid helper DOPE, also dedicated to nucleic acid delivery, were evaluated for protein transfer ( $\beta$-gal, antibodies, DNA methyl transferase (MT))[99]. They proved to be more efficient than BioPorter ${ }^{\circledR}[96]$, for $\beta$ gal and antibody cytoplasmic delivery in presence of serum. Interestingly, SAINT:DOPE liposomes also allow nuclear delivery and functionality of MT. Surprisingly, the size of the formed complexes was not correlated to the initial protein size. Indeed, $\beta$-gal (116 kDa) gave $138 \mathrm{~nm}$ complexes whereas MT (45 kDa) led to larger complexes (1753 nm). Zeta potential also varied from positively charged, neutral to negatively charged. The structure, hydrophilic/lipophilic profile of the protein was therefore reported to strongly impact the physico-chemical profile of these complexes. More recently, Chatin et al. evaluated different nucleic acid dedicated reagent to vectorize proteins and evidenced the necessity to adapt the lipid assortment to the vectorised protein [100]. Interestingly, the couple associating bis (guanidinium)-tren-cholesterol (BGTC) as a cationic lipid and DOPE as a helper lipid was efficient to allow $\beta$-gal cytoplasmic delivery, but inefficient for antibody targeting cytokeratin K (K8) delivery. By contrast, K8 was fully internalised into $70 \%$ of HeLa cells thanks to dioleyl succinyl paromomycin (DOSP) as a cationic lipid and an imidazole based helper lipid (MM27). To be able to complex proteins, the lipid/protein ratio ranged from 600 to 2500 for $\beta$-gal and from 200 to 1500 for $\mathrm{K} 8$, forming complexes in the micrometre range $(1-5 \mu \mathrm{m})$ mainly dedicated to in vitro use. Altogether, the last two mentioned studies confirmed that protein-complex properties are poorly predictable as they strongly depends on protein associated size, charge and conformation. Unlike nucleic acids which expose the same structure and charge independently of their sequence, proteins vary in structure, charge and hydrophilic profile, and these parameters the development of a versatile protein vector.

To circumvent aforementioned well-known drawbacks of cationic lipids, including poor in vivo efficiency, cationic lipidoids were developed by Anderson and Langer [101]. High throughput cationic lipid screening was therefore performed to find optimised candidate molecules. A major difference between thus developed lipid-like molecules (lipidoids) is that they are generally not structured as classic cationic lipids composed of a cationic head and lipidic tails, but generally expose less distinct/binary structures. These cationic lipids 
formulated or not with other lipids, have proven effective in delivering a wide range of cargo, mainly nucleic acids, but also proteins. Indeed, this screening approach is particularly relevant to adress concerns of protein heterogeneity and to identify one or more lipid(s) which can best adapt to the structure of each individual protein. In 2014, the first lipidoid library dedicated to protein cytoplasmic delivery was created by synthesizing a 1,2-epoxyhexadecane tail structure on 14 different aliphatic amine core structures [102]. To allow protein complexation, proteins were modified with cis-aconitic anhydride (protein-Aco), thus reinforcing electrostatic interactions between protein and cationic lipids. The modification was shown to be reversible when performed with RNase A in slightly acidic endolysosomal-like environment (evaluated at $\mathrm{pH}$ 5.2), restoring active protein. Delivery and action of RNase A and Sap were evaluated into murine melanoma B16F10 cells. Among evaluated lipids, EC16-1 was the most efficient to deliver RNase A-Aco, Sap and Sap-Aco and allow cell viability reduction to $30 \%$. Whereas Sap could be delivered without modification, RNase A without Aco modification could not be, probably due to the positive charge and hydrophilic nature of RNase A which poorly interact with hydrophobic positively charged lipids. Sap was formulated in lipid NPs composed of EC16-1, DOPE, cholesterol, ceramide-mPEG 2000 to allow in vivo IV administration in BALB/c mice bearing 4T1 breast cancer tumours. 28 days post-injection, tumour volumes were significantly reduced compared to free Sap (by $80 \%$ ) with no side effects (no body weight changes, no increase in TNF- $\alpha$ or IFN- $\gamma$ levels). This efficiency was confirmed more recently in vivo in two models of colon (SW620/AD300) and lung (NCIH460/MX20) carcinoma multidrug resistance tumours [103].

In a follow-up study, RNAse A lysine's residues were modified using 4-nitrophenyl 4-(4,4,5,5-tetramethyl-1,3,2-dioxaborolan-2-yl) benzyl carbonate (NBC) to trigger ROS responsive release of RNAse A and reduce protein charge to allow complexation with cationic entities [104]. Another member of EC16 lipids (EC16-80) was evidenced as efficient for RNase-NBC complexation. Such RNase A-NBC loaded lipid complexes were able to decrease cell viability to at least $40 \%$ in a panel of cancer cell lines (HeLa cervical cancer cells, B16F10 melanoma cells, PC-3 prostate cancer cells, and MDA-MB-231 breast cancer cells), but not in non-cancer cells suggesting that the ROS level, increased in cancer cells, could led to a ROS responsive protein cytotoxic activity. The same lipid (EC16-80) was used to complex tumor suppressor protein PTEN, and led, in vitro to a decrease in cell viability to $20 \%$ into PC3 cells [105]. Delivered PTEN negatively regulated the AKT pathway and induce apoptosis (20\% of annexin V positive PC3 cells after PTEN/EC16-80 treatment). Nevertheless, the physicochemical characterization of such complexes, not associated with 
stabilizing lipids, revealed a relatively high zeta potential $(+48 \mathrm{mV})$, which could explain the absence of in vivo evaluation of such formulations for now. In parallel, a new library of 12 bio-reducible, GSH sensitive lipids were evaluated to complex genome editing proteins [106]. Synthesized lipids were composed of (i) different head groups with primary and/or secondary amines, (ii) an acrylate that features a disulfide bond and (iii) two 14-carbon hydrophobic tails. Among 5 efficient lipids, one was selected to deliver negatively charged Cas9-RNP complexes since it had efficiency similar to commercially available lipids to target and inhibit genomic EGFP reporter gene expression into HEK cell line (70 \% EGFP expression loss). Thanks to bioreducible disulfide bonds in lipids, the intracellular dissociation of complexes was favored, enabling nucleus delivery. Still to deliver Cas9-RNP, a library of chalcogencontaining lipids was created by reacting lipophilic tails containing $\mathrm{O}, \mathrm{S}$ or Se ethers (named O17O, O17S or O17Se) with various amine heads [107]. One lipid (81-O17Se) was particularly interesting to allow Cas9-RNP delivery in reporter GFP-HEK cell line, resulting in GFP knockout levels comparable to commercial standards (50\% vs. 63\%) but with lower cytotoxicity ( $24 \%$ vs. $34 \%$ ). These results, while encouraging, will have to be completed with findings exhibiting therapeutic potential.

\subsubsection{Non cationic lipid-based systems}

Non cationic EC16 derived lipidoids dedicated to protein delivery were recently developed using nitrilotriacetic acid NTA groups, associated with divalent nickel ions and helper lipids (cholesterol, phospholipids, and PEGylated lipids) [108]. His-tagged-NLS-Cas9RNP/lipidoid complexes formed well dispersed 150-350 nm nanoparticles. Already evocated GFP-HEK cell line was used to evaluate the genome editing efficiency, leading to GFP KO inhibition in the range of $15 \%$, an lower amount compared to the one obtained with GSH sensitive cationic lipidoids systems previously developed [106]. Nevertheless, this study established the proof-of-concept that non cationic lipidoids can be used to load and deliver Cas9-RNP complexes. Further optimisation of the synthesis of lipidoids will undoubtedly lead to increased efficiency in the future.

As an innovative source of lipid, a mix of neutral to negatively charged lipids isolated from spinach chloroplast thylacoids was used to form small unilamellar liposomes [109]. In parallel, mitochondrial membrane protein VDAC (voltage dependent anion channel) and proapoptotic Bak protein were produced in cell-free manner using Rapid Translation System (RTS) relying on Escherichia coli lysate to perform coupled in vitro transcription-translation reactions. Liposomes were "simply" added to the RTS mix before $48 \mathrm{~h}$ incubation. After 
purification, Bak, VDAC or Bak-VDAC loaded liposomes, called proteoliposomes were used to treat HCT116 colon cancer cells. After $24 \mathrm{~h}, 20 \%$ of treated cells were annexin V positive vs. $8.5 \%$ for unloaded liposome and $48 \%$ for doxorubicin treated cells). Cas-3 assay also revealed a slight increase in Cas3 positive cell after Bak liposome $(22.25 \%)$ treatment compared to other formulations or unloaded liposomes $(2.7 \%)$. Even if no control using free proteins were performed for these functional assays, the study was original in term of protein as well as lipid source. Moreover, it presents the advantage of being possible to perform in one step. A pro-apoptotic effect was also evidenced into GL26 glioblastoma cells treated with Bak loaded proteoliposomes using a different lipid source but the same loading strategy [110]. In vivo, this formulation induced tumour regression in $40 \%$ of mice carrying ectopic GBM model (GL26) after intratumoural injection. Systemic administration also led to an increased tumour cell apoptosis and animal life span, but only a slight delay in tumour outgrow, maybe due to a lack of efficiency to reach tumour cells.

PC:Chol liposomes were used by Mastrobattista et al. to co-encapsulate diphtheria toxin A chain (DTA) $(70 \mathrm{kDa})$ and an endosome fusogenic peptide resembling the $\mathrm{N}$-terminal domain of influenza virus hemagglutinin (diINF-7) [111]. DTA was associated to such liposomes by rehydration of PC:Chol lipid film. The formed liposomes were then extruded onto polycarbonate membrane (till $0.05 \mu \mathrm{m}$ ) to obtain $193 \mathrm{~nm}$ particles. Liposomes, functionalised with DSPE-mPEG 2000 attached to a mAb targeting EGFR were used to treat ovarian carcinoma cell line (OVCAR-3) over -expressing EGFR. Interestingly, the cytoplasmic delivery of diINF-7/DTA-containing immunoliposomes (DTA-425-FIL) led to 8fold higher cytotoxicity compared to free DTA, or liposomes loaded with only diINF-7 or DTA. These results showed that intracellular delivery of DTA into OVCAR-3 cells required both receptor-mediated endocytosis of DTA/diINF-7 immunoliposomes as well as subsequent activation of the endosomolytic activity of diINF-7 in low $\mathrm{pH}$ environment. Unfortunately, to our knowledge, this promising system has not been evaluated in vivo yet.

\subsubsection{Hybrid lipid based systems}

Recently, a library of new lipid-containing oligoaminoamides was synthesized to allow cas9-RNP complexation by electrostatic interactions [112]. Interestingly, the presence of one hydroxyl group in the fatty acid was evidenced as crucial to form stable well-defined nanoparticles and increase cellular uptake, endosomal escape as well as nuclear delivery. A GFP reporter gene was used as a read-out to assess the knock out efficiency of cas9RNP targeting this gene. The optimised construct gave 40\% GFP knock out in Neuro2A-GFP cells 
after a single Cas9/sgRNA treatment. After a proof-of-concept in artificial reporter cell lines, this system was also evaluated to knock out an endogenous gene, the folate receptor 1 gene. Into Hela cell line, FolR 1 knock out of around 30\% were detected. These promising results now call for formulation optimisation adapted to in vivo evaluation.

\section{Conclusion}

In response to a strong therapeutic need, many systems have been developed in the last decade to allow protein intracellular delivery. The major challenge in this research area, is the complexity of proteins, which prevents the development of a reference system applicable to the majority of proteins and implies a case-by-case pharmaceutical engineering. As evidenced in the present review, the majority of nanoparticles used to complex proteins are cationic systems (lipid or polymer or modified inorganic NP). This imply, if proteins to deliver are not naturally negatively charged, to use biological or chemical modification to allow electrostatic interactions. Such modifications could hinder biological functions of proteins, increase system complexity and are not applicable to all proteins. Some transient modifications (cis A in particular) made it possible to overcome this drawback, as attested by the development of promising systems.

Compare systems in term of efficiencies is not an easy task, as studies differ in protein cargo, as well as efficiencies assays, or target cells. In the last year, the discovery of a powerful molecular tool, CRISPR-Cas9, has called for the development of a multitude of vectors capable of co-delivering several biomolecules in the same device. We therefore have chosen to compare systems able to deliver such editing tools, as an example of a cargo delivered by various nanocarriers (both organic and inorganic). To be efficient, CRISPR-Cas 9 protein should be associated to a single guide RNA (sgRNA) to form RiboNucleoProtein (RNP) complexes and, if a specific edition is wanted, a DNA template/donor (containing the desired sequence to be edited bordered by homologous sequences named homology arms) should be provided. CRISP-cas9 can be used to create gene Knock out (KO) by forming a double stranded break (DSB) at the DNA target site followed by repair of this break by nonhomologous end-joining (NHEJ) pathway (Figure 5). NHEJ will induce insertion of small nucleotide insertion or deletion (indel) at the DSB site leading to mutation inducing premature stop codon and loss of function of the target gene. CRISPR-cas9 can also be used to accurately edit gene by after DSB by Homology directed repair (HDR) if a DNA repair 
template is provided. Nevertheless, the percentage of effective HDR are generally low (inf to $10 \%$ ). The majority of DSB will therefore be repaired by NHJE. Among studies evocated in this work, CRISPR-cas 9 efficiency was measured regarding mainly KO efficiencies, and, for only 3 studies, regarding gene edition by HDR (Table 5). The whole cas9-RNP complex is negatively charged, thus allowing complexation with cationic systems (cationic polymer, cationic lipids as well as AuNP modified with positive charges). Among systems evaluated in this work, two were polymer based NP [75, 88], three were Au based NP [35, 42, 43], and five were lipid based NP [95, 106-108, 113]. In vitro KO efficiencies until 80\% were obtained for polymer and lipid based systems (Table 5). By contrast, Au based systems led to 40-50\% $\mathrm{KO}$ efficiencies for the most efficient systems. Among the three systems evaluated in vivo, similar in vivo KO efficiencies were obtained (ranging from 8 to 13\%). All systems were used to deliver cas9 RNP complex while only 2 systems allow concomitant delivery of donor DNA [35, 113]: Lipofectamine ${ }^{\circledR}$ CRISPRMAX were able to induce $17 \%$ HDR (in vitro in HEK293 cells), while obtained HDR were from 3 to $11 \%$ (for endogenous gene or eGFP repair reporter respectively) with CRISPR-Gold complexes [35]. These systems therefore exposed comparable efficiencies to induce HDR in vitro. Only Gold based systems were shown to induce HDR in vivo by restoring dystrophin gene after intramuscular administration.

It is difficult to select a system that could be specifically highlighted to be the most efficient for CRISPR cas9 delivery system. Nevertheless, lipid based nanoparticles, mainly based on cationic lipid use, confirmed their status of leader nanocarriers for protein delivery, especially CRISPR Cas9-RNP complexes, with 5 different lipid-based formulations dedicated to this transfer, and biological activities among the most efficient in selected studies. The domain of inorganic nanoparticles, particularly AuNPs, especially when associated in a complex multi-materials systems, such as the one developed by Lee et al. [35] (Figure 5E), associating AuNP, silica structure and cationic polymer, led to delivery efficiencies also among the best, even in vivo after local administration. This type of hybrid system, by selecting advantages of both inorganic and organic systems, are probably a good example of future research trends, to elaborate systems able to respond to the requirement of protein cytoplasmic delivery. To date, few systems have been assessed in vivo and none of them, to our knowledge, has reached clinical trials so far. Nevertheless, as the development of protein intracellular transfer method is a major challenge, boosted by the discovery of gene editing tools, there is no doubt that such systems will reach the pre-clinic and clinic soon. 


\section{Bibliography}

1. Leader, B., Q.J. Baca, and D.E. Golan, Protein therapeutics: a summary and pharmacological classification. Nat Rev Drug Discov, 2008. 7(1): p. 21-39.

2. Usmani, S.S., et al., THPdb: Database of FDA-approved peptide and protein therapeutics. PLoS One, 2017. 12(7): p. e0181748.

3. Urquhart, L., Market watch: Top drugs and companies by sales in 2017. Nat Rev Drug Discov, 2018. 17(4): p. 232.

4. Leader, B., Q.J. Baca, and D.E. Golan, Protein therapeutics: A summary and pharmacological classification. Nature Reviews Drug Discovery, 2008. 7(1): p. 21-39.

5. Strohl, W.R., Current progress in innovative engineered antibodies. Protein \& cell, 2018. 9(1): p. 86-120.

6. Miersch, S. and S.S. Sidhu, Intracellular targeting with engineered proteins. F1000Research, 2016. 5.

7. Vazquez-Lombardi, R., et al., Challenges and opportunities for non-antibody scaffold drugs. Drug Discovery Today, 2015. 20(10): p. 1271-1283.

8. Yu, M.Y., et al., Nanotechnology for protein delivery: Overview and perspectives. Journal of Controlled Release, 2016. 240: p. 24-37.

9. Chakrabarti, R., D.E. Wylie, and S.M. Schuster, Transfer of monoclonal antibodies into mammalian cells by electroporation. The Journal of biological chemistry, 1989. 264(26): p. 15494-500.

10. Keppeke, G.D., et al., Microinjection of specific anti-IMPDH2 antibodies induces disassembly of cytoplasmic rods/rings that are primarily stationary and stable structures. Cell \& bioscience, 2015. 5(1): p. 1.

11. Togtema, M., et al., Sonoporation delivery of monoclonal antibodies against human papillomavirus 16 E6 restores p53 expression in transformed cervical keratinocytes. PloS one, 2012. 7(11): p. e50730.

12. Wen, R., et al., Intracellular Delivery and Sensing System Based on Electroplated Conductive Nanostraw Arrays. Acs Applied Materials \& Interfaces, 2019. 11(47): p. 43936-43948.

13. Chen, Y.P., et al., Cellular Deformations Induced by Conical Silicon Nanowire Arrays Facilitate Gene Delivery. Small, 2019. 15(47).

14. Chen, Y.P., et al., Silicon-Nanotube-Mediated Intracellular Delivery Enables Ex Vivo Gene Editing. Advanced Materials, 2020. 32(24).

15. Lestrell, E., et al., Engineered nano-bio interfaces for intracellular delivery and sampling: Applications, agency and artefacts. Materials Today, 2020. 33: p. 87-104.

16. Chiappini, C., et al., Biodegradable silicon nanoneedles delivering nucleic acids intracellularly induce localized in vivo neovascularization. Nature Materials, 2015. 14(5): p. 532-539.

17. Bolhassani, A., B.S. Jafarzade, and G. Mardani, In vitro and in vivo delivery of therapeutic proteins using cell penetrating peptides. Peptides, 2017. 87: p. 50-63.

18. Kristensen, M., D. Birch, and H.M. Nielsen, Applications and Challenges for Use of CellPenetrating Peptides as Delivery Vectors for Peptide and Protein Cargos. International Journal of Molecular Sciences, 2016. 17(2).

19. Zeng, B.R., H.D. Shi, and Y.Z. Liu, A versatile pH-responsive platform for intracellular protein delivery using calcium phosphate nanoparticles. Journal of Materials Chemistry B, 2015. 3(47): p. 9115-9121.

20. Boussif, O., et al., A versatile vector for gene and oligonucleotide transfer into cells in culture and in vivo: polyethylenimine. Proc Natl Acad Sci U S A, 1995. 92(16): p. 7297-301.

21. Das, P. and N.R. Jana, Length-Controlled Synthesis of Calcium Phosphate Nanorod and Nanowire and Application in Intracellular Protein Delivery. Acs Applied Materials \& Interfaces, 2016. 8(13): p. 8710-8720.

22. Li, J., et al., Biodegradable calcium phosphate nanoparticle with lipid coating for systemic siRNA delivery. Journal of Controlled Release, 2010. 142(3): p. 416-421. 
23. Fang, Y., et al., Lipid-Coated, pH-Sensitive Magnesium Phosphate Particles for Intracellular Protein Delivery. Pharm Res, 2019. 36(6): p. 81.

24. Vallet-Regi, M., et al., Mesoporous Silica Nanoparticles for Drug Delivery: Current Insights. Molecules, 2018. 23(1): p. 19.

25. Alhmoud, H., et al., Porous Silicon Nanodiscs for Targeted Drug Delivery. Advanced Functional Materials, 2015. 25(7): p. 1137-1145.

26. Alhmoud, H., et al., A MACEing silicon: Towards single-step etching of defined porous nanostructures for biomedicine. Progress in Materials Science, 2021. 116.

27. Han, D.H., et al., Direct cellular delivery of human proteasomes to delay tau aggregation. Nature Communications, 2014. 5: p. 8.

28. Xu, C., et al., Core-Cone Structured Monodispersed Mesoporous Silica Nanoparticles with Ultra-large Cavity for Protein Delivery. Small, 2015. 11(44): p. 5949-5955.

29. Meka, A.K., et al., A Vesicle Supra-Assembly Approach to Synthesize Amine-Functionalized Hollow Dendritic Mesoporous Silica Nanospheres for Protein Delivery. Small, 2016. 12(37): p. 5169-5177.

30. Yang, Y.N., et al., Structure-Dependent and Glutathione-Responsive Biodegradable Dendritic Mesoporous Organosilica Nanoparticles for Safe Protein Delivery. Chemistry of Materials, 2016. 28(24): p. 9008-9016.

31. Shao, D., et al., Bioinspired Diselenide-Bridged Mesoporous Silica Nanoparticles for DualResponsive Protein Delivery. Advanced Materials, 2018. 30(29).

32. Bale, S.S., et al., Nanoparticle-Mediated Cytoplasmic Delivery of Proteins To Target Cellular Machinery. Acs Nano, 2010. 4(3): p. 1493-1500.

33. Niu, Y.T., et al., Understanding the contribution of surface roughness and hydrophobic modification of silica nanoparticles to enhanced therapeutic protein delivery. Journal of Materials Chemistry B, 2016. 4(2): p. 212-219.

34. Zhang, J., et al., Synthesis of Silica Vesicles with Controlled Entrance Size for High Loading, Sustained Release, and Cellular Delivery of Therapeutical Proteins. Small, 2014. 10(24): p. 5068-5076.

35. Lee, K., et al., Nanoparticle delivery of Cas9 ribonucleoprotein and donor DNA in vivo induces homology-directed DNA repair. Nature Biomedical Engineering, 2017. 1(11): p. 889901.

36. Khan, A.K., et al., Gold Nanoparticles: Synthesis and Applications in Drug Delivery. Tropical Journal of Pharmaceutical Research, 2014. 13(7): p. 1169-1177.

37. Ghosh, P., et al., Intracellular Delivery of a Membrane-Impermeable Enzyme in Active Form Using Functionalized Gold Nanoparticles. Journal of the American Chemical Society, 2010. 132(8): p. 2642-2645.

38. Tang, R., et al., Direct Delivery of Functional Proteins and Enzymes to the Cytosol Using Nanoparticle-Stabilized Nanocapsules. Acs Nano, 2013. 7(8): p. 6667-6673.

39. Tang, R., et al., Cytosolic delivery of large proteins using nanoparticle-stabilized nanocapsules. Nanoscale, 2016. 8(42): p. 18038-18041.

40. Mout, R. and V.M. Rotello, A General Method for Intracellular Protein Delivery through 'Etag' Protein Engineering and Arginine Functionalized Gold Nanoparticles. Bio-Protocol, 2017. 7(24)

41. Mout, R. and V.M. Rotello, Cytosolic and Nuclear Delivery of CRISPR/Cas9ribonucleoprotein for Gene Editing Using Arginine Functionalized Gold Nanoparticles. BioProtocol, 2017. 7(20).

42. Lee, Y.W., et al., In Vivo Editing of Macrophages through Systemic Delivery of CRISPRCas9-Ribonucleoprotein-Nanoparticle Nanoassemblies. Advanced Therapeutics, 2019. 2(10).

43. Ju, E.G., et al., Gold Nanocluster-Mediated Efficient Delivery of Cas9 Protein through pHInduced Assembly-Disassembly for Inactivation of Virus Oncogenes. Acs Applied Materials \& Interfaces, 2019. 11(38): p. 34717-34724.

44. Ryou, S.-M., et al., Gold nanoparticle-DNA aptamer composites as a universal carrier for in vivo delivery of biologically functional proteins. Journal of Controlled Release, 2014. 196: p. 287-294. 
45. Yeom, J.H., et al., Intracellular delivery of recombinant proteins via gold nanoparticle-DNA aptamer composites is independent of the protein physicochemical properties and cell type. Journal of Industrial and Engineering Chemistry, 2017. 45: p. 5-10.

46. Chen, Y.-S., et al., Assessment of the In Vivo Toxicity of Gold Nanoparticles. Nanoscale Research Letters, 2009. 4(8): p. 858-864.

47. Balasubramanian, S.K., et al., Biodistribution of gold nanoparticles and gene expression changes in the liver and spleen after intravenous administration in rats. Biomaterials, 2010. 31(8): p. 2034-2042.

48. Balfourier, A., et al., Unexpected intracellular biodegradation and recrystallization of gold nanoparticles. Proceedings of the National Academy of Sciences of the United States of America, 2020. 117(1): p. 103-113.

49. Furukawa, H., et al., The Chemistry and Applications of Metal-Organic Frameworks. Science, 2013. 341(6149): p. 974-+.

50. Wu, M.X. and Y.W. Yang, Metal-Organic Framework (MOF)-Based Drug/Cargo Delivery and Cancer Therapy. Advanced Materials, 2017. 29(23): p. 20.

51. Deng, H.X., et al., Large-Pore Apertures in a Series of Metal-Organic Frameworks. Science, 2012. 336(6084): p. 1018-1023.

52. Liang, K., et al., Biomimetic mineralization of metal-organic frameworks as protective coatings for biomacromolecules. Nature Communications, 2015. 6.

53. Roder, R., et al., Multifunctional Nanoparticles by Coordinative Self-Assembly of His Tagged Units with Metal Organic Frameworks. Journal of the American Chemical Society, 2017. 139(6): p. 2359-2368.

54. Lian, X.Z., et al., High efficiency and long-term intracellular activity of an enzymatic nanofactory based on metal-organic frameworks. Nature Communications, 2017. 8.

55. Chen, T.T., et al., Biomineralized Metal-Organic Framework Nanoparticles Enable Intracellular Delivery and Endo-Lysosomal Release of Native Active Proteins. Journal of the American Chemical Society, 2018. 140(31): p. 9912-9920.

56. Cheng, G., et al., Self-Assembly of Extracellular Vesicle-like Metal-Organic Framework Nanoparticles for Protection and Intracellular Delivery of Biofunctional Proteins. Journal of the American Chemical Society, 2018. 140(23): p. 7282-7291.

57. Kam, N.W.S. and H.J. Dai, Carbon nanotubes as intracellular protein transporters: Generality and biological functionality. Journal of the American Chemical Society, 2005. 127(16): p. 6021-6026.

58. Kam, N.W.S., et al., Nanotube molecular transporters: Internalization of carbon nanotubeprotein conjugates into mammalian cells. Journal of the American Chemical Society, 2004. 126(22): p. 6850-6851.

59. Li, H., X.Q. Fan, and X. Chen, Near-Infrared Light Activation of Proteins Inside Living Cells Enabled by Carbon Nanotube-Mediated Intracellular Delivery. Acs Applied Materials \& Interfaces, 2016. 8(7): p. 4500-4507.

60. Lacerda, S.H.D., et al., Carbon Nanotubes Activate Store-Operated Calcium Entry in Human Blood Platelets. Acs Nano, 2011. 5(7): p. 5808-5813.

61. Zhang, J.X., M. Zheng, and Z.G. Xie, Co-assembled hybrids of proteins and carbon dots for intracellular protein delivery. Journal of Materials Chemistry B, 2016. 4(34): p. 5659-5663.

62. Li, J., et al., An intracellular protein delivery platform based on glutathione-responsive protein nanocapsules. Chemical Communications, 2016. 52(93): p. 13608-13611.

63. Gu, Z., et al., Protein Nanocapsule Weaved with Enzymatically Degradable Polymeric Network. Nano Letters, 2009. 9(12): p. 4533-4538.

64. Biswas, A., et al., Endoprotease-Mediated Intracellular Protein Delivery Using Nanocapsules. Acs Nano, 2011. 5(2): p. 1385-1394.

65. Zhao, M.X., et al., Redox-responsive nanocapsules for intracellular protein delivery. Biomaterials, 2011. 32(22): p. 5223-5230.

66. Biswas, A., et al., Polyethylene glycol-based protein nanocapsules for functional delivery of a differentiation transcription factor. Biomaterials, 2012. 33(21): p. 5459-5467.

67. Zhao, M.X., et al., Clickable Protein Nanocapsules for Targeted Delivery of Recombinant p53 Protein. Journal of the American Chemical Society, 2014. 136(43): p. 15319-15325. 
68. Liu, M., et al., Hierarchical Nanoassemblies-Assisted Combinational Delivery of Cytotoxic Protein and Antibiotic for Cancer Treatment. Nano Letters, 2018. 18(4): p. 2294-2303.

69. Lamb, R., et al., Antibiotics that target mitochondria effectively eradicate cancer stem cells, across multiple tumor types: Treating cancer like an infectious disease. Oncotarget, 2015. 6(7): p. 4569-4584.

70. Al-Hajj, M., et al., Prospective identification of tumorigenic breast cancer cells. Proceedings of the National Academy of Sciences of the United States of America, 2003. 100(7): p. 39833988.

71. Jiang, Y., et al., Protein Toxin Chaperoned by LRP-1-Targeted Virus-Mimicking Vesicles Induces High-Efficiency Glioblastoma Therapy In Vivo. Advanced Materials, 2018. 30(30).

72. Fan, K.L., et al., Ferritin Nanocarrier Traverses the Blood Brain Barrier and Kills Glioma. Acs Nano, 2018. 12(5): p. 4105-4115.

73. Ran, D.N., et al., GRP78 enabled micelle-based glioma targeted drug delivery. Journal of Controlled Release, 2017. 255: p. 120-131.

74. Singhal, A., et al., Nanoparticle-mediated catalase delivery protects human neurons from oxidative stress. Cell Death \& Disease, 2013. 4.

75. Rui, Y., et al., Carboxylated branched poly(beta-amino ester) nanoparticles enable robust cytosolic protein delivery and CRISPR-Cas9 gene editing. Science Advances, 2019. 5(12).

76. Zhang, X.J., K. Zhang, and R. Haag, Multi-stage, charge conversional, stimuli-responsive nanogels for therapeutic protein delivery. Biomaterials Science, 2015. 3(11): p. 1487-1496.

77. Kawasaki, R., et al., Magnetically Guided Protein Transduction by Hybrid Nanogel Chaperones with Iron Oxide Nanoparticles. Angewandte Chemie-International Edition, 2016. 55(38): p. 11377-11381.

78. Chen, J., et al., EGFR and CD44 Dual-Targeted Multifunctional Hyaluronic Acid Nanogels Boost Protein Delivery to Ovarian and Breast Cancers In Vitro and In Vivo. Acs Applied Materials \& Interfaces, 2017. 9(28): p. 24140-24147.

79. Hu, Q.Z., et al., Complete regression of breast tumour with a single dose of docetaxelentrapped core-cross-linked polymeric micelles. Biomaterials, 2015. 53: p. 370-378.

80. Hrkach, J., et al., Preclinical Development and Clinical Translation of a PSMA-Targeted Docetaxel Nanoparticle with a Differentiated Pharmacological Profile. Science Translational Medicine, 2012. 4(128).

81. Chen, J., et al., Saporin-loaded CD44 and EGFR dual-targeted nanogels for potent inhibition of metastatic breast cancer in vivo. International Journal of Pharmaceutics, 2019. 560: p. 5764.

82. Huang, K., et al., Small, Traceable, Endosome-Disrupting, and Bioresponsive Click Nanogels Fabricated via Microfluidics for CD44-Targeted Cytoplasmic Delivery of Therapeutic Proteins. Acs Applied Materials \& Interfaces, 2019. 11(25): p. 22171-22180.

83. Lee, Y., et al., Charge-Conversional Polyionic Complex Micelles-Efficient Nanocarriers for Protein Delivery into Cytoplasm. Angewandte Chemie-International Edition, 2009. 48(29): p. 5309-5312.

84. Lee, Y., et al., Efficient Delivery of Bioactive Antibodies into the Cytoplasm of Living Cells by Charge-Conversional Polyion Complex Micelles. Angewandte Chemie-International Edition, 2010. 49(14): p. 2552-2555.

85. Kim, A., et al., Intracellular Delivery of Charge-Converted Monoclonal Antibodies by Combinatorial Design of Block/Homo Polyion Complex Micelles. Biomacromolecules, 2016. 17(2): p. 446-453.

86. Qiu, M., et al., Small-Sized and Robust Chimaeric Lipopepsomes: A Simple and Functional Platform with High Protein Loading for Targeted Intracellular Delivery of Protein Toxin in Vivo. Chemistry of Materials, 2018. 30(19): p. 6831-6838.

87. Lv, J., et al., Polymers for cytosolic protein delivery. Biomaterials, 2019. 218.

88. Liu, C.Y., et al., A boronic acid-rich dendrimer with robust and unprecedented efficiency for cytosolic protein delivery and CRISPR-Cas9 gene editing. Science Advances, 2019. 5(6).

89. Okuro, K., et al., Molecular Glues Carrying Multiple Guanidinium Ion Pendants via an Oligoether Spacer: Stabilization of Microtubules against Depolymerization. Journal of the American Chemical Society, 2009. 131(5): p. 1626-+. 
90. Meuzelaar, H., M.R. Panman, and S. Woutersen, Guanidinium-Induced Denaturation by Breaking of Salt Bridges. Angewandte Chemie-International Edition, 2015. 54(50): p. 1525515259.

91. Chang, H., et al., Rational Design of a Polymer with Robust Efficacy for Intracellular Protein and Peptide Delivery. Nano Letters, 2017. 17(3): p. 1678-1684.

92. Martins, S., et al., Lipid-based colloidal carriers for peptide and protein delivery--liposomes versus lipid nanoparticles. International journal of nanomedicine, 2007. 2(4): p. 595-607.

93. Bardania, H., S. Tarvirdipour, and F. Dorkoosh, Liposome-targeted delivery for highly potent drugs. Artificial Cells, Nanomedicine, and Biotechnology, 2017. 45(8): p. 1478-1489.

94. Zuris, J.A., et al., Cationic lipid-mediated delivery of proteins enables efficient protein-based genome editing in vitro and in vivo. Nature Biotechnology, 2015. 33(1): p. 73-80.

95. $\mathrm{Yu}, \mathrm{X}$., et al., Improved delivery of Cas 9 protein/gRNA complexes using lipofectamine CRISPRMAX. Biotechnology Letters, 2016. 38(6): p. 919-929.

96. Zelphati, O., et al., Intracellular delivery of proteins with a new lipid-mediated delivery system. Journal of Biological Chemistry, 2001. 276(37): p. 35103-35110.

97. Zheng, X.Y., et al., Lipid-mediated protein delivery of suicide nucleoside kinases. Cancer Research, 2003. 63(20): p. 6909-6913.

98. Dalkara, D., G. Zuber, and J.P. Behr, Intracytoplasmic delivery of anionic proteins. Molecular Therapy, 2004. 9(6): p. 964-969.

99. van der Gun, B.T.F., et al., Serum insensitive, intranuclear protein delivery by the multipurpose cationic lipid SAINT-2. Journal of Controlled Release, 2007. 123(3): p. 228-238.

100. Chatin, B., et al., Liposome-based Formulation for Intracellular Delivery of Functional Proteins. Molecular Therapy-Nucleic Acids, 2015. 4.

101. Akinc, A., et al., A combinatorial library of lipid-like materials for delivery of RNAi therapeutics. Nature Biotechnology, 2008. 26(5): p. 561-569.

102. Wang, M., et al., Combinatorially Designed Lipid-like Nanoparticles for Intracellular Delivery of Cytotoxic Protein for Cancer Therapy. Angewandte Chemie-International Edition, 2014. 53(11): p. 2893-2898.

103. Zhang, G.N., et al., Lipid-Saporin Nanoparticles for the Intracellular Delivery of Cytotoxic Protein to Overcome ABC Transporter-Mediated Multidrug Resistance In Vitro and In Vivo. Cancers, 2020. 12(2).

104. Wang, M., et al., Reactive Oxygen Species-Responsive Protein Modification and Its Intracellular Delivery for Targeted Cancer Therapy. Angewandte Chemie-International Edition, 2014. 53(49): p. 13444-13448.

105. Altinoglu, S.A., et al., Intracellular delivery of the PTEN protein using cationic lipidoids for cancer therapy. Biomaterials Science, 2016. 4(12): p. 1773-1780.

106. Wang, M., et al., Efficient delivery of genome-editing proteins using bioreducible lipid nanoparticles. Proceedings of the National Academy of Sciences of the United States of America, 2016. 113(11): p. 2868-2873.

107. Li, Y.M., et al., Combinatorial library of chalcogen-containing lipidoids for intracellular delivery of genome-editing proteins. Biomaterials, 2018. 178: p. 652-662.

108. Li, Y.M., A.C. Li, and Q.B. Xu, Intracellular Delivery of His-Tagged Genome-Editing Proteins Enabled by Nitrilotriacetic Acid-Containing Lipidoid Nanoparticles. Advanced Healthcare Materials, 2019. 8(6): p. 10.

109. Liguori, L., et al., Liposomes-mediated delivery of pro-apoptotic therapeutic membrane proteins. Journal of Controlled Release, 2008. 126(3): p. 217-227.

110. Liguori, L., et al., Anti-Tumor Effects of Bak-Proteoliposomes against Glioblastoma. Molecules, 2015. 20(9): p. 15893-15909.

111. Mastrobattista, E., et al., Functional characterization of an endosome-disruptive peptide and its application in cytosolic delivery of immunoliposome-entrapped proteins. Journal of Biological Chemistry, 2002. 277(30): p. 27135-27143.

112. Kuhn, J., et al., Delivery of Cas9/sgRNA Ribonucleoprotein Complexes via Hydroxystearyl Oligoamino Amides. Bioconjugate Chemistry, 2020. 31(3): p. 729-742.

113. Zuris, J.A., et al., Cationic lipid-mediated delivery of proteins enables efficient protein-based genome editing in vitro and in vivo. Nature Biotechnology, 2015. 33(1): p. 73-80. 


\section{FIGURES}

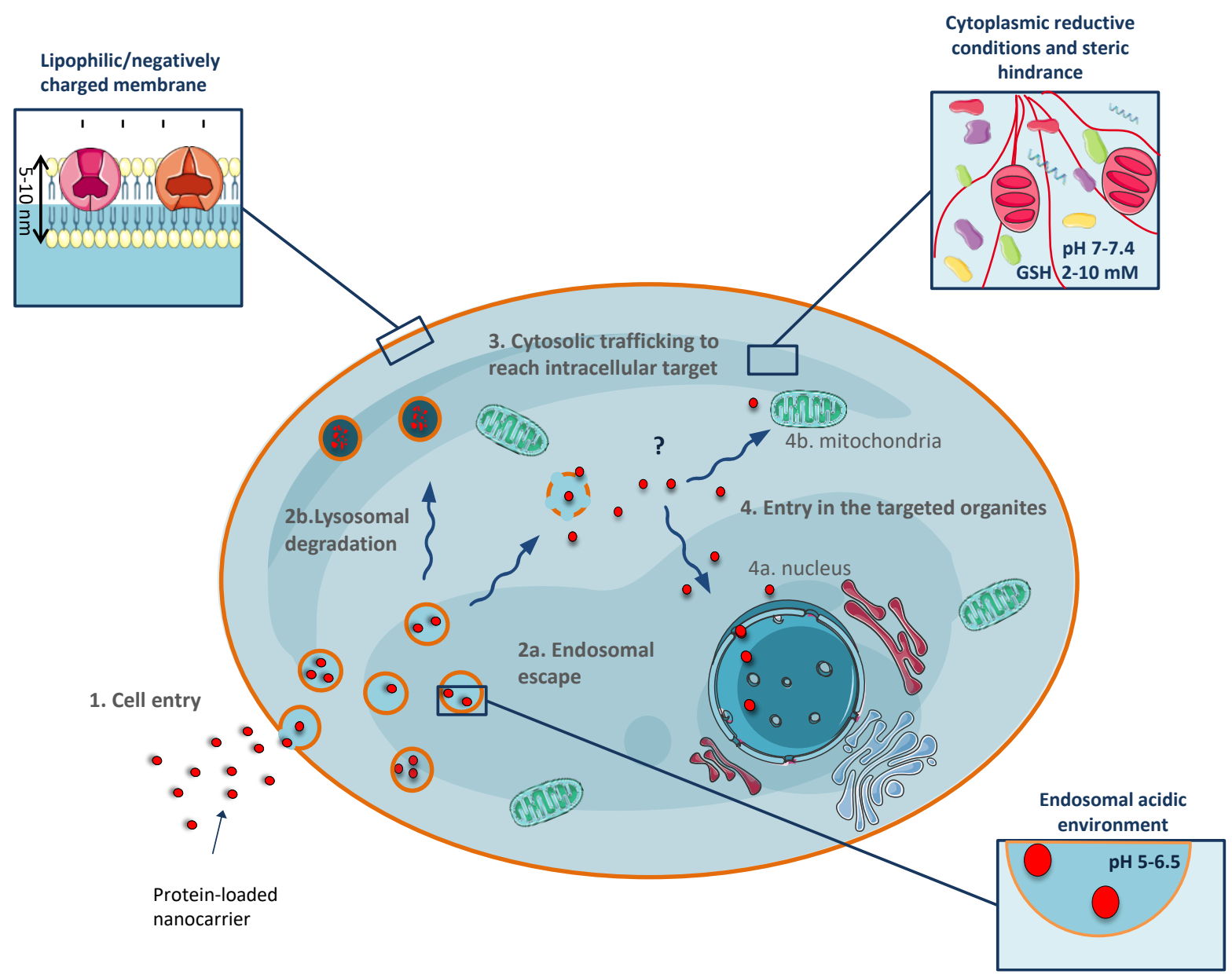

Figure 1. Barriers encountered by protein nanocarriers to achieve intracellular delivery. Protein loaded nanocarriers have to enter the cell (1). This entry is generally mediated by endocytosis followed by retention into endosomal vesicles. Then, the cargo protein has to escape exosomes (2a) or it will be degraded into lysosomes (2b). At this step, nanocarrier and protein could be still associated or dissociated, depending on systems. Afterwards, the protein has to traffic into reductive and molecular crowded cytoplasm to reach its target (3), and if required, depending on the protein action mechanism, to enter specific organelles, such as nucleus (4a) or mitochondria (4b). 


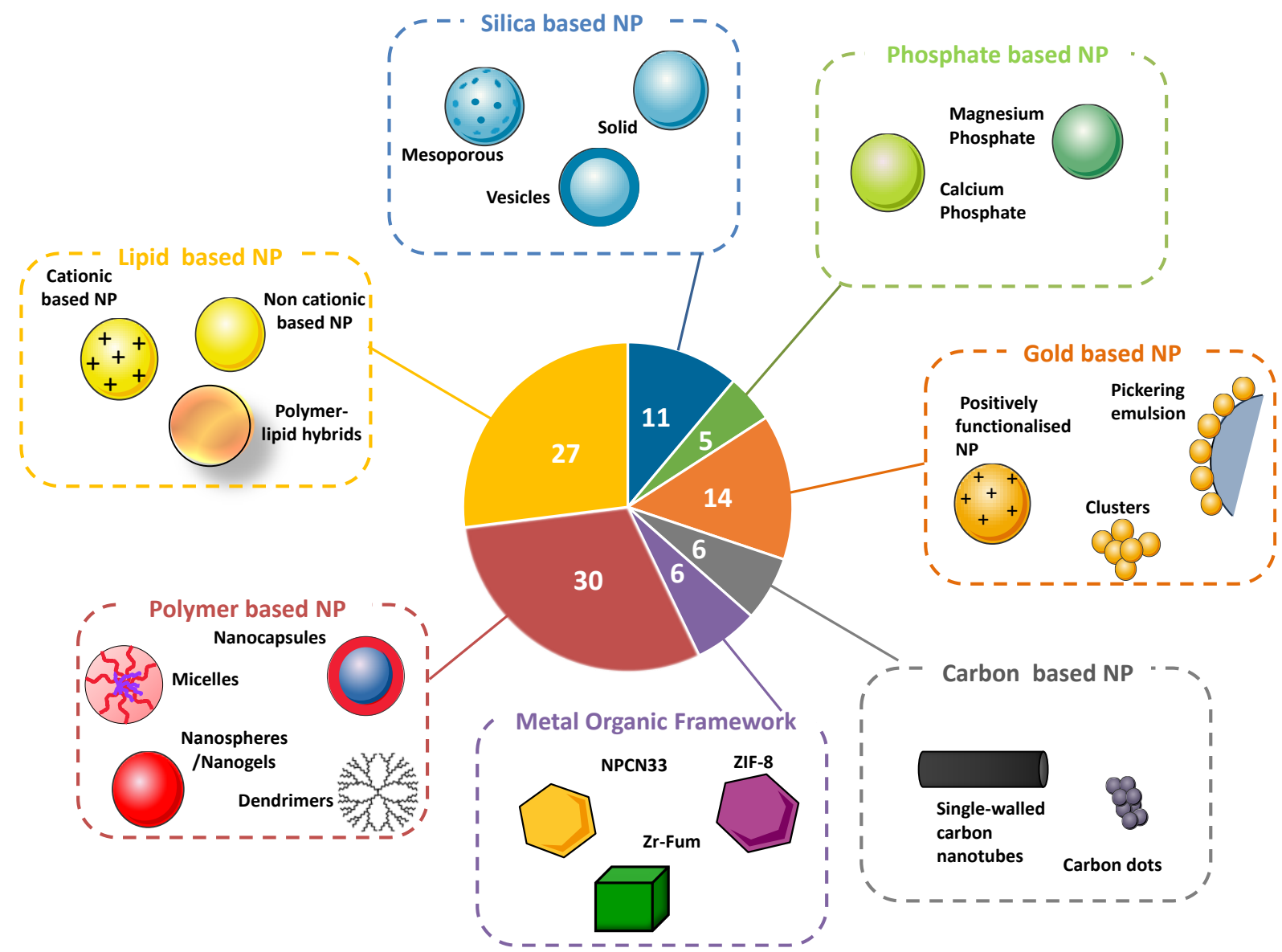

Figure 2. Nanocarriers dedicated to protein intracellular delivery described in this manuscript represented in percentage $(n=63)$. Among these studies, several systems were investigated for protein intracellular delivery: silica based nanoparticle (NP) $(n=7)$, phosphate based NP ( $n=3)$, Au based NP (n=9), Metal Organic Framework $(n=4)$, carbon based NP $(n=4)$, polymer based NP $(n=19)$, and lipid based NP $(n=17)$. 
AuNP - HKRK pep

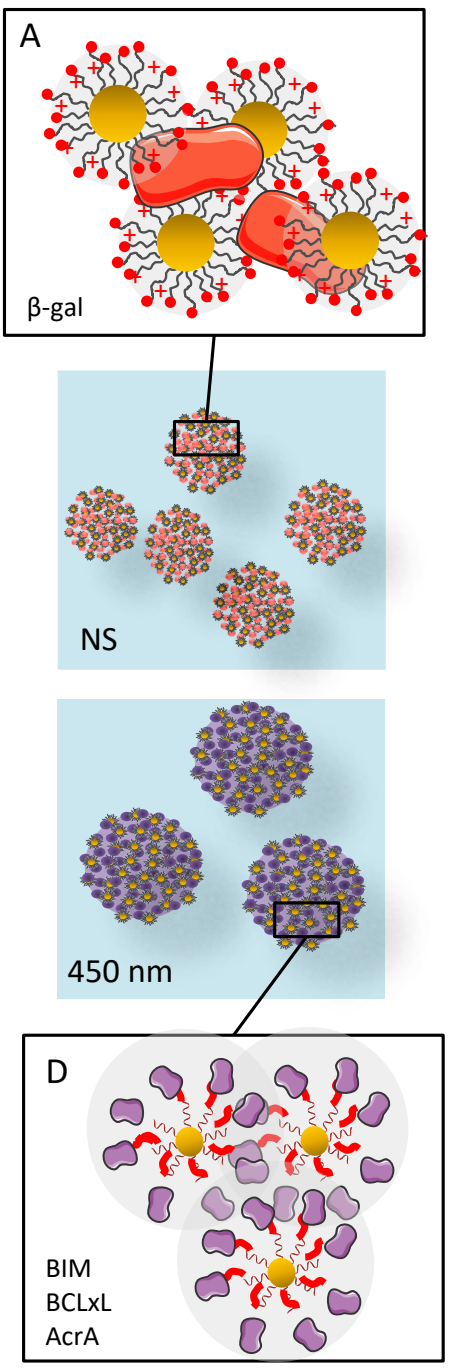

AuNP-Apt anti His

+ His-tagged prot
Nanoparticle-stabilised capsules (NPSCs)
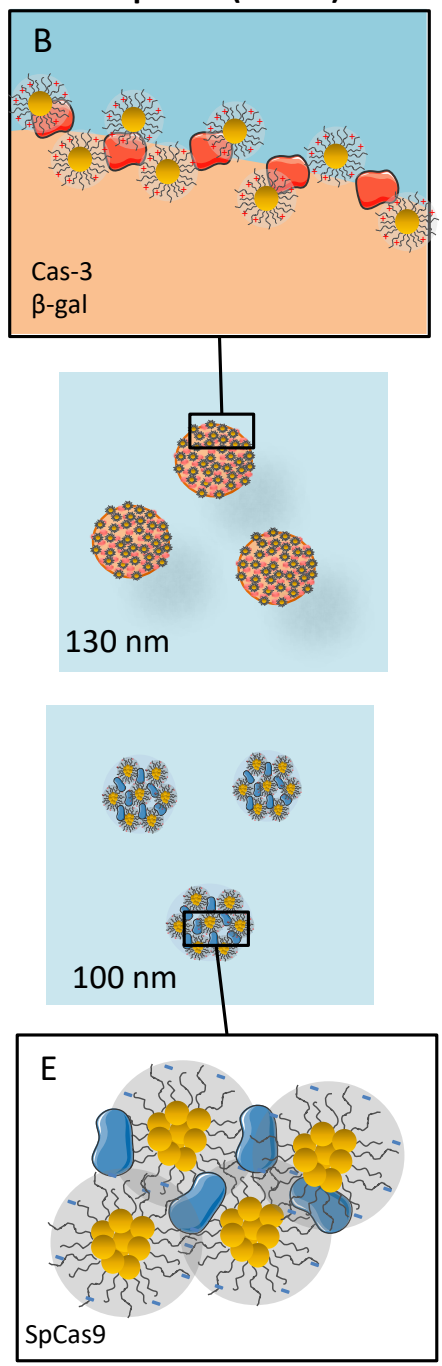

AuNC-GSH
AuNP - Arg

+ (E-tag) protein

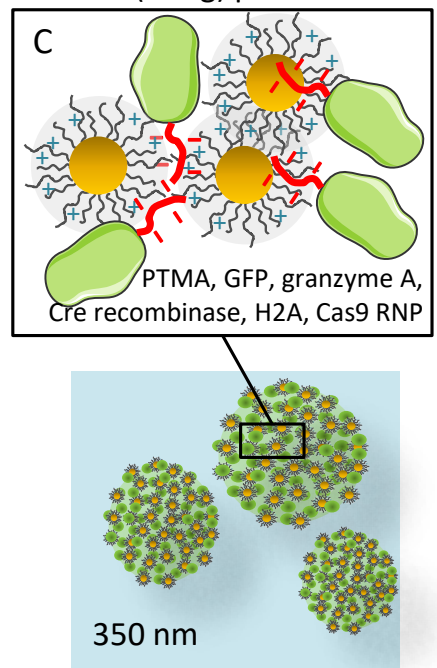

NS
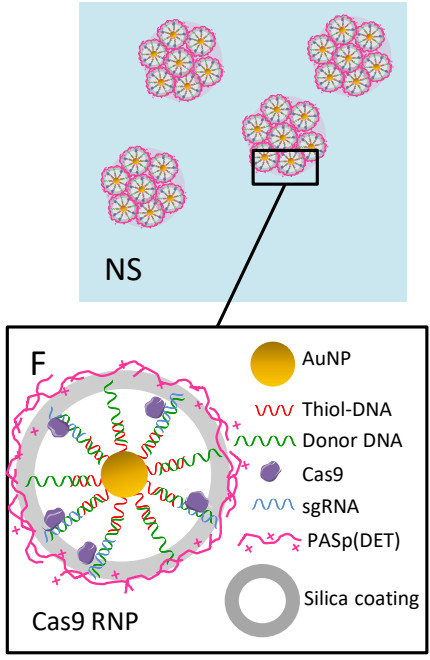

CRISPR-Gold

Figure 3. Schematic representation of different strategies relying on Au NP assemblies with proteins. A. AuNP (13nm) - HKRK pep[37]; NS: non specified. B. Nanoparticle-stabilised capsules (NPSCs): Au nNPs (2nm) functionalized with HKRK peptides used to stabilise linoleic acid-based nano-emulsions, so-called Pickering- emulsions [38, 39]. C. AuNP (2 nm)-Arg + oligo-glutamic acid negative tagged (E-tag) protein [40, 41]. D. AuNP-Apt anti His [44, 45]. E. Au Nanoclusters functionnalised with gluthation moieties (GSH) (negatively charged at physiological pH to interact with positively charged SpCas9) [43]. F. AuNPs were first functionalised with 5' thiol modified single-stranded DNA to complex donor DNA template through complementary base-pairing. Cas9-RNP was then added to the construct and a coating with silica was performed to increase stability. Finally, an endosomal disruptive polymer (poly $(N-[N-(2$-aminoethyl)-2aminoethyl]aspartamide) (PAsp(DET)) was added [35]. 


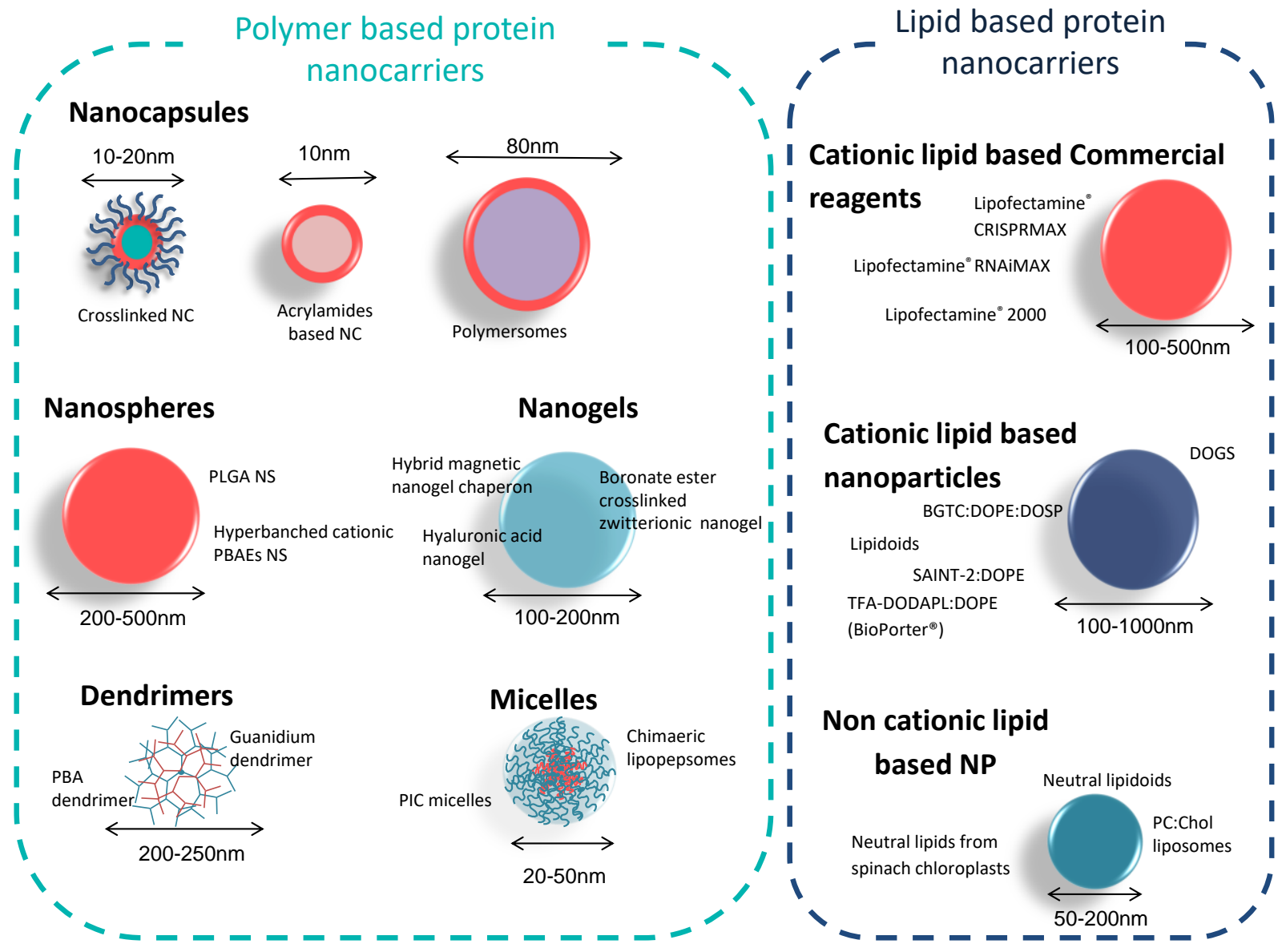

Figure 4. Schematic representation of organic nanocarriers. PBA: phenylboronic acid; PBAEs: $\operatorname{poly}(\beta-$ amino ester); PIC: polyanion complex; PLGA: poly(lactic co-glucolic acid; DOGS: dioctadecylglycylspermine; DOPE: dioleoyl phosphatidylethanolamine; DOPS:1,2-Dioleoyl-sn-glycero-3phospho-L-serine; DOSP: dioleyl succinyl paromomycin; SAINT-2: N-methyl-4(dioleyl)methyl-pyridiniumchlorideTFA-DODAPL: trifluoroacetylated lipopolyamine 


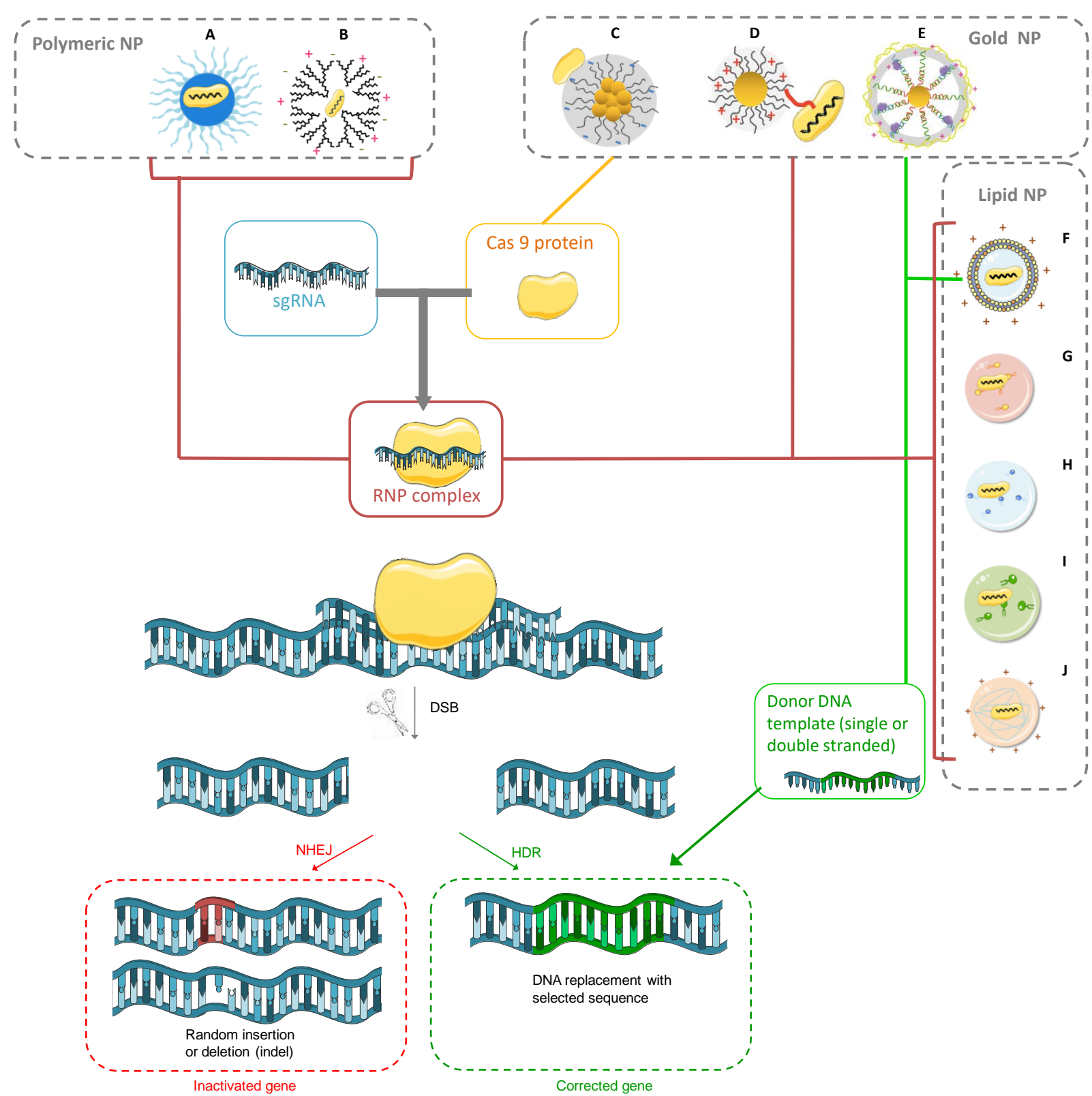

Figure 5. Schematic representation of different nanocarriers used for CRISPR Cas9 machinery delivery.

A. Hyperbranched cationic PBAEs NS [75]. B. PBA modified PAMAM dendrimers (100nm) [88]. C. Au Nanoclusters functionalized with gluthation moities (GSH) [43]. D. AuNP (2 nm)-Arg + oligo-glutamic acid negative tagged (E-tag) protein [41]. E. AuNPs (15nm) - functionalized with 5' thiol modified single-stranded DNA, coated with silica [35]. F. Cationic lipid based commercial reagents: Lipofectamine ${ }^{\circledR}$ RNAiMAX and 2000 [89] and Lipofectamine CRISPRMAX [95]. G. Lipidoids with primary and/or secondary amines heads, acrylate disulfide bond and 14-carbon hydrophobic tails [106]. H. Chalcogen-containing lipidoid [107]. I. Non cationic lipidoid [108]. J. Lipid-containing oligoaminoamides[112]. Abbreviations. Cas9: CRISPR-associated protein 9; PBA: phenylboronic acid; PBAEs: poly( $\beta$-amino ester)s; RNP: ribonucleoprotein. To allow gene edition via CRISPR Cas9 machinery, three actors should be transferred into cell nucleus. The first actor is the non-specific endonuclease protein Cas9. To allow matching to a specific target on gene, a single stranded RNA guide (sgRNA) is necessary to direct Cas9 to a specific gene location. This sgRNA is generally composed of a $5^{\prime}$ 17-20-nucleotide sequence complementary to the target DNA sequence and of a $3^{\prime}$ sequence necessary to bind to Cas9 protein. The assembly of protein Cas9 and sgRNA gives a negatively charged complexe named 
RiboNucleoProtein complex (Cas9-RNP). This complex is therefore able to find, match to its target sequence where it will be capable to cut the double stranded DNA thus inducing a double-strand break (DSB). At this stage, DNA repair mechanisms, including non-homologous end joining (NHEJ) and homology-directed repair (HDR), are activated. The NHEJ pathway could cause mutational insertions and deletions (Indels) of short sequences at the repair site, causing disruption of the target gene. If an exogenous donor DNA template with sequence homology to the target site is provided, an HDR could enables targeted donor DNA insertion at the Cas9 cut site for specific gene correction or addition. 


\section{TABLES}

Table 1. Silica based NP for protein intracellular delivery. Abbreviations: MSNP : mesoporous silica nanoparticles; NP : nanoparticles; : IgG : immunoglobuline G; RITC: rhodamine $\mathrm{B}$ isothiocyanate labelled ; $\beta$-gal: $\beta$-galactosidase; RNase A : ribonuclease $\mathrm{A} ; \mathrm{CytC}$ : cytochrome $\mathrm{C}$.

\begin{tabular}{|c|c|c|c|c|c|c|c|}
\hline Structure & Particle description & Schematic structure & $\begin{array}{l}\text { Particle } \\
\text { size }\end{array}$ & Pore size & Protein (size) & $\begin{array}{c}\text { Biological } \\
\text { application }\end{array}$ & References \\
\hline \multirow{5}{*}{ MESOPOROUS } & $\begin{aligned} & \text { MSNP }-\mathrm{Ni}^{2+} \\
+ & \text { His tagged protein }\end{aligned}$ & & $\begin{array}{c}239 \pm 27 \\
\mathrm{~nm}\end{array}$ & $25-30 \mathrm{~nm}$ & $\begin{array}{l}\beta 4 \text { proteasome } \\
\text { subunit }(29 \mathrm{kDa})\end{array}$ & In vitro & [27] \\
\hline & Core-cone MSNP & & $\begin{array}{l}220-261 \\
\mathrm{~nm}\end{array}$ & $3-46 \mathrm{~nm}$ & $\begin{array}{l}\text { IgG -RITC (150 } \\
\text { kDa) } \\
\beta \text {-gal }(119 \mathrm{kDa})\end{array}$ & In vitro & [28] \\
\hline & $\begin{array}{c}\text { Amine-hollow dendritic } \\
\text { MSNP }\end{array}$ & & $\begin{array}{c}360 \pm 37 \\
\mathrm{~nm}\end{array}$ & $21 \mathrm{~nm}$ & $\begin{array}{l}\text { IgG }(150 \mathrm{kDa}) \beta \text {-gal } \\
\text { tetramer }(465 \mathrm{kDa})\end{array}$ & In vitro & [29] \\
\hline & $\begin{array}{c}\text { Dendritic MSNP + GSH } \\
\text { sens moieties ( } \mathrm{S}-\mathrm{S} \text { bonds) }\end{array}$ & & $220 \mathrm{~nm}$ & 4- $14 \mathrm{~nm}$ & $\begin{array}{l}\text { RNAse A-Aco (13.7 } \\
\text { kDa) }\end{array}$ & In vitro & {$[30]$} \\
\hline & $\begin{array}{l}\text { Diselenide-bridged MSNP } \\
+ \text { cell membran coating }\end{array}$ & & $\begin{array}{c}120-190 \\
\mathrm{~nm}\end{array}$ & $\begin{array}{c}8.5-11.3 \\
\mathrm{~nm}\end{array}$ & $\begin{array}{l}\text { RNAse A }(13.7 \\
\text { kDa) }\end{array}$ & $\begin{array}{l}\text { In vitro } \\
\text { In vivo }\end{array}$ & {$[31]$} \\
\hline VESICLE & Silica vesicles & & $50 \mathrm{~nm}$ & $3.9-34 \mathrm{~nm}$ & $\begin{array}{c}\text { CytC } \\
\text { RNAse A }(13.7 \\
\text { kDa })\end{array}$ & In vitro & {$[34]$} \\
\hline SOLID & $\begin{array}{l}\text { Solid silica NP + } \\
\text { hydrophobic coating } \\
\text { + roughness }\end{array}$ & & $\begin{array}{c}286-306 \\
n m\end{array}$ & $\mathrm{x}$ & $\begin{array}{l}\text { RNAse A }(13.7 \\
\mathrm{kDa})\end{array}$ & In vitro & {$[32]$} \\
\hline
\end{tabular}


Table 2. MOF nanoparticle protein delivery systems. Abbreviations. $\beta$-gal: $\beta$-galactosidase; BSA: bovine serum albumin; Cas-3: caspase-3; CAT: catalase; CytC: cytochrome C; GFP: green fluorescent protein; MOF: metal-organic frameworks; RFP: red fluorescent protein; SOD: super oxide dismutase

\begin{tabular}{|c|c|c|c|c|c|}
\hline MOF nanoparticles & Metal cluster & Organic ligands & $\begin{array}{c}\text { Associated protein } \\
\text { (protein size) }\end{array}$ & $\begin{array}{c}\text { Biological } \\
\text { application }\end{array}$ & Ref. \\
\hline Zr-fum & Zirconium & Furamic acid & CytC (12 kDa) & In vitro & [53] \\
\hline NPCN-333 & Aluminium trimetric & Triaminotrinitrobenzene & SOD (17 kDa); CAT (60 kDa) & In vitro & [54] \\
\hline \multirow[t]{2}{*}{ ZIF-8 } & Zinc & 2-methylimidazole & $\begin{array}{c}\text { BSA }(60 \mathrm{kDa}) ; \text { eGFP }(27 \mathrm{kDa}) ; \beta \text {-gal }(116 \mathrm{kDa}) \text {; } \\
\text { Cas3 }(31 \mathrm{kDa}) / \mathrm{BSA} ; \mathrm{BSA} / \mathrm{RFP}(26 \mathrm{kDa}) / \beta \text {-gal; Anti } \\
\text { tubulin antibody }(52 \mathrm{kDa})\end{array}$ & In vitro & [55] \\
\hline & Zinc & 2-methylimidazole & BSA; gelonin (28 kDa) & In vitro / in vivo & [56] \\
\hline
\end{tabular}


Table 3. Polymeric nanoparticle protein delivery systems. Abbreviations. $\beta$-gal: $\beta$-galactosidase; BSA: bovine serum albumin; Cas-3: caspase-3; Cas9: CRISPRassociated protein 9; CAT: catalase; CLP: chimaeric lipopepsomes; Cyt-C: cytochrome C; GrB: granzyme B; GFP: green fluorescent protein; HRP: horse radish peroxidase IgG: immunoglobin G Klf4: Kruppel-like factor 4; NC: nanocapsule; NS: nanosphere; PBA: phenylboronic acid; PBAEs: poly( $\beta$-amino ester); PIC: polyanion complex; PLGA: poly(lactic co-glucolic acid; RFP: red fluorescent protein; RNase A: ribonuclease A; R-PE: R-phycoerythrin; Sap: saporin; YFP: Yellow fluorescent protein.

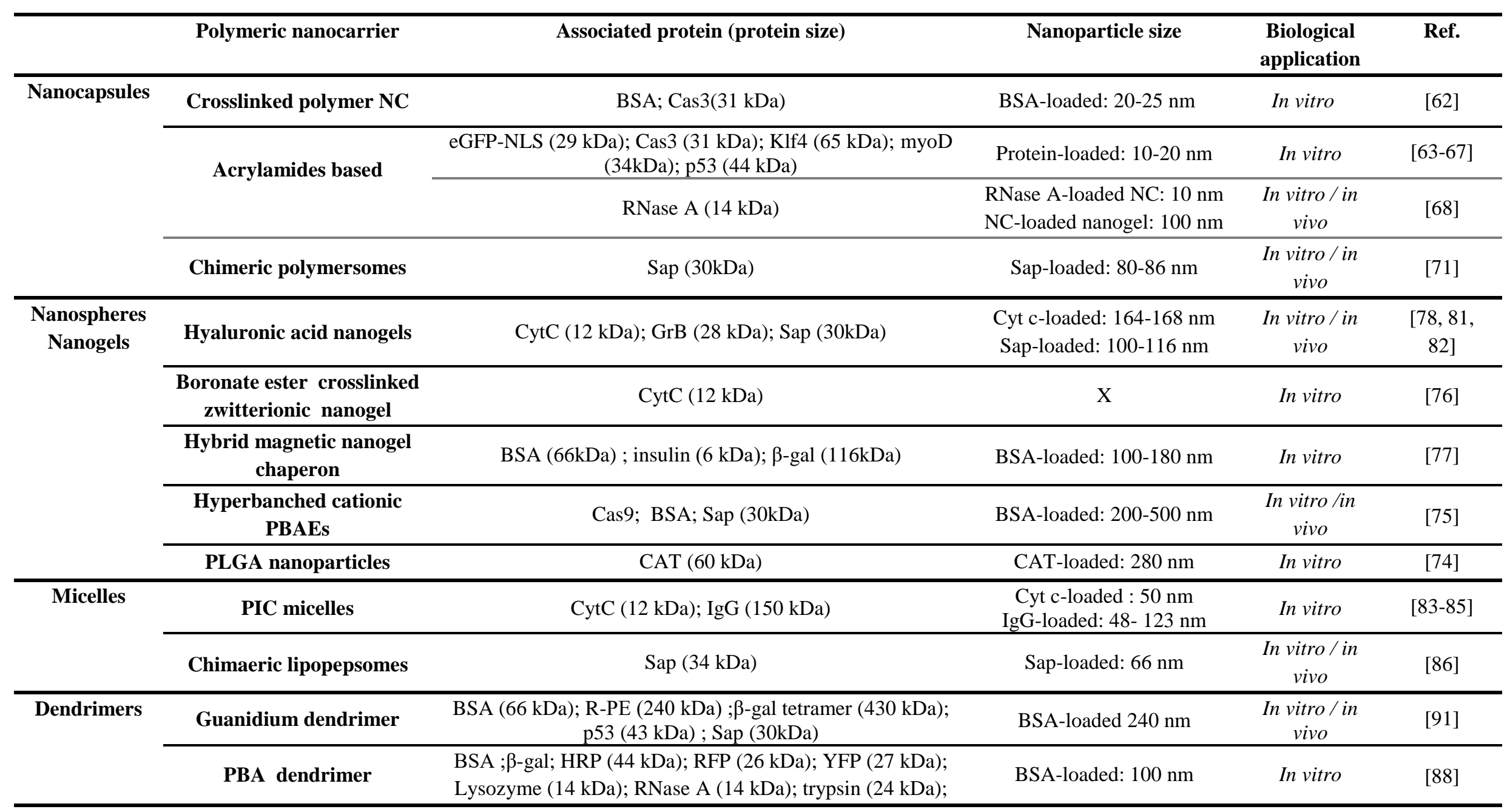


Table 4. Lipid nanoparticle protein delivery systems. Abbreviations. Ab: antibodies ; $\beta$-gal: $\beta$-galactosidase; BGTC: bis (guanidinium)-tren-cholesterol; BSA: bovine serum albumin; Cas-3: caspase-3; Cas-8: caspase-8; Cas9: CRISPR-associated protein 9; Chol: cholesterol; Cyt-C: cytochrome C; DMPE-PEG 2000 : 1,2-dimyristoyl-snglycero-3-phosphoethanolamine-N-[methoxy(polyethylene glycol)-2000]; DOGS: dioctadecylglycylspermine; DOPE: dioleoyl phosphatidylethanolamine; DOPS:1,2Dioleoyl-sn-glycero-3-phospho-L-serine; DOSP: dioleyl succinyl paromomycin; DTA: diphtheria toxin A; FITC: fluorescein isothiocynate; GrB: granzyme B; IgG: immunoglobin G; NTA: nitrilotriacetic acid; PC: phosphatidylcholine; PTEN: tumor suppressor protein; RNase A: ribonuclease A; RNPs: ribonucleoproteins; R-PE: Rphycoerythrin; SAINT-2: $N$-methyl-4(dioleyl)methyl-pyridinium-chloride; TFA-DODAPL: trifluoroacetylated lipopolyamine; VDAC: voltage dependent anion channel.

\begin{tabular}{|c|c|c|c|c|}
\hline Formulation & Lipid composition & Associated protein (protein size) & $\begin{array}{l}\text { Biological } \\
\text { application }\end{array}$ & Ref. \\
\hline \multirow{2}{*}{$\begin{array}{l}\text { Cationic lipid } \\
\text { based } \\
\text { commercial } \\
\text { reagents }\end{array}$} & Lipofectamine® RNAiMAX and 2000 & Cas9-RNPs $(\sim 160 \mathrm{kDa})$ & In vitro and in vivo & {$[113]$} \\
\hline & Lipofectamine $®$ CRISPRMAX & Cas9-RNPs ( 160 kDa) & In vitro & {$[95]$} \\
\hline \multirow{7}{*}{$\begin{array}{l}\text { Cationic lipid } \\
\text { based } \\
\text { nanoparticles }\end{array}$} & TFA-DODAPL:DOPE (BioPorter®) & $\begin{array}{c}\text { Fluorescently tagged Ab; phycoerythrin; Cas-3 (31 kDa); Cas-8 (55 } \\
\mathrm{kDa}) ; \mathrm{GrB}(28 \mathrm{kDa}) ; \beta \text {-gal }(116 \mathrm{kDa}) ; \mathrm{CytC}(12 \mathrm{kDa}) ; \text { protein } \\
\text { nucleoside kinase }(17 \mathrm{kDa})\end{array}$ & In vitro & {$[96,97]$} \\
\hline & DOGS & 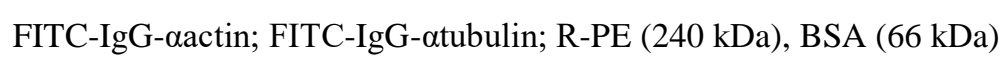 & In vitro & {$[98]$} \\
\hline & SAINT-2:DOPE & $\begin{array}{c}\beta \text {-gal }(116 \mathrm{kDa}) \\
\text { DNA methyl transferase }(45 \mathrm{kDa})\end{array}$ & In vitro & [99] \\
\hline & $\begin{array}{c}\text { BGTC:DOPE } \\
\text { DOSP: imidazole based }\end{array}$ & $\begin{array}{c}\beta \text {-gal }(116 \mathrm{kDa}) \\
\text { Antibody targeting cytokeratin } \mathrm{K}\end{array}$ & In vitro & {$[100]$} \\
\hline & Lipidoid EC16-1 ; EC16-80 & RNase A (14 kDa); saporin (34 kDa); PTEN (47kDa) & In vitro and in vivo & [102-105] \\
\hline & $\begin{array}{l}\text { Primary and/or secondary amines heads with acrylate } \\
\text { disulfide bond and two 14-carbon hydrophobic tails }\end{array}$ & Cas9-RNPs ( 160 kDa) & In vitro & {$[106]$} \\
\hline & $\begin{array}{l}\text { Chalcogen-containing lipids with lipophilic tails and } \\
\text { amines }\end{array}$ & Cas9-RNPs $(\sim 160 \mathrm{kDa})$ & In vitro & [107] \\
\hline
\end{tabular}




\begin{tabular}{|c|c|c|c|c|}
\hline \multirow{3}{*}{$\begin{array}{l}\text { Non cationic } \\
\text { lipid based }\end{array}$} & $\begin{array}{l}\text { Lipidoid composed of NTA groups, divalent nickel } \\
\text { ions and helper lipids (cholesterol, phospholipid) }\end{array}$ & His-tagged Cas9-RNPs ( 160 kDa) & In vitro & [108] \\
\hline & $\begin{array}{l}\text { Neutral to negatively charged lipids from spinach } \\
\text { chloroplast thylacoids }\end{array}$ & Pro-apoptotic Bak protein $(60 \mathrm{kDa})$; VDAC $(32 \mathrm{kDa})$ & In vitro and in vivo & $\begin{array}{l}{[109,} \\
110]\end{array}$ \\
\hline & PC:Chol liposomes & $\begin{array}{l}\text { DTA (70 kDa) co-encapsulated with influenza-derived fusogenic } \\
\text { peptide }\end{array}$ & In vitro & [111] \\
\hline $\begin{array}{l}\text { Hybrid lipid } \\
\text { based }\end{array}$ & Lipid-containing oligoaminoamides & Cas9-RNPs ( 160 kDa) & In vitro & [112] \\
\hline
\end{tabular}


Table 5. nanoparticle based systems for gene editing protein (Cas9-RNP) delivery. Second column of Table 5 refer to schematic representation of these systems in Fig. 5. Abbreviations. Cas9-RNP : Caspase 9-sgRNA Ribonucleoprotein complex; HDR (homology-directed repair); iv : intravenous; PBAEs : hyperbranched cationic poly( $\beta$-amino ester)s; PBA: phenylboronic acid.

\begin{tabular}{|c|c|c|c|c|c|}
\hline NP type & $\begin{array}{l}\text { Fig.5 } \\
\text { ID }\end{array}$ & Formulation & Evaluation & Efficiency test/Main results & Ref. \\
\hline \multirow{2}{*}{$\begin{array}{l}\text { Polymer } \\
\text { based NP }\end{array}$} & A & Hyperbanched cationic PBAEs NS & In vitro & $\begin{array}{l}80 \% \mathrm{KO} \text { targeted gene (GFP) } \\
\text { HDR in vitro : } 4 \%\end{array}$ & [75] \\
\hline & B & PBA modified PAMAM dendrimers & In vitro & $40 \% \mathrm{KO}$ targeted gene (GFP) & {$[88]$} \\
\hline \multirow{3}{*}{$\begin{array}{l}\text { Au based } \\
\text { NP }\end{array}$} & $\mathrm{C}$ & $\begin{array}{c}\text { Au Nanoclusters functionnalized with gluthation } \\
\text { moities (GSH) }\end{array}$ & In vitro & $50 \% \mathrm{KO}$ targeted gene (HPV18 E6 oncogene) & [43] \\
\hline & $\mathrm{D}$ & $\begin{array}{c}\text { AuNP (2nm)-Arg + oligo-glutamic acid negative } \\
\text { tagged (E-tag) protein }\end{array}$ & In vitro/in vivo & $\begin{array}{l}\text { In vitro : } 40 \% \mathrm{KO} \text { targeted gene (GFP) } \\
\text { In vivo (iv) : } 8 \% \text { KO targeted gene (PTEN) }\end{array}$ & {$[41,42]$} \\
\hline & E & $\begin{array}{l}\text { AuNPs }(15 \mathrm{~nm}) \text { - functionalised with } 5 \text { ' thiol } \\
\text { modified single-stranded DNA, coated with silica }\end{array}$ & In vitro & $\begin{array}{c}\text { In vitro: } 3-11 \% \text { HDR (eGFP reporter or } \\
\text { endogenous gene) } \\
\text { In vivo : } 5 \% \text { HDR (dystrophin gene) }\end{array}$ & [35] \\
\hline \multirow{5}{*}{$\begin{array}{l}\text { Liposomes/ } \\
\text { Lipid NP }\end{array}$} & $\mathrm{F}$ & Lipofectamine ${ }^{\circledR}$ RNAiMAX and 2000 & In vitro /in vivo & $\begin{array}{l}\text { In vitro : } 60-80 \% \text { gene } \mathrm{KO} \text { (eGFP) } \\
8-11 \% \mathrm{HDR}(\mathrm{eGFP} \text { repair reporter) } \\
\text { In vivo (local) }: 13 \% \mathrm{KO} \text { (eGFP) }\end{array}$ & [113] \\
\hline & G & Lipofectamine ${ }^{\circledR}$ CRISPRMAX & In vitro & $\begin{array}{c}\text { In vitro : 8\% (MCF-7 cells) to 85\% (HEK293) \% } \\
\text { indels } \\
\text { HDR in HEK293 : 17\% HDR }\end{array}$ & [95] \\
\hline & $\mathrm{H}$ & Cationic bioreducible lipids & In vitro & $70 \% \mathrm{KO}$ targeted gene (eGFP) & [106] \\
\hline & I & Chalcogen containing lipidoids & In vitro & $50 \% \mathrm{KO}$ targeted gene (eGFP) & [107] \\
\hline & $\mathrm{J}$ & Non cationic lipid based & In vitro & $15 \% \mathrm{KO}$ targeted gene (eGFP) & {$[108]$} \\
\hline
\end{tabular}


